\title{
Physics of reshock and mixing in single-mode Richtmyer-Meshkov instability
}

\author{
Oleg Schilling* \\ University of California, Lawrence Livermore National Laboratory, Livermore, California 94551, USA
}

Marco Latini ${ }^{\dagger}$

Applied and Computational Mathematics, California Institute of Technology, Pasadena, California 91125, USA

Wai Sun Don

Division of Applied Mathematics, Brown University, Providence, Rhode Island 02912, USA

(Received 13 December 2006; published 30 August 2007)

\begin{abstract}
The ninth-order weighted essentially nonoscillatory (WENO) shock-capturing method is used to investigate the physics of reshock and mixing in two-dimensional single-mode Richtmyer-Meshkov instability to late times. The initial conditions and computational domain were adapted from the Mach 1.21 air (acetone)/SF 6 shock tube experiment of Collins and Jacobs [J. Fluid Mech. 464, 113 (2002)]: the growth of the bubble and spike amplitudes from fifth- and ninth-order WENO simulations of this experiment were compared to the predictions of linear and nonlinear amplitude growth models, and were shown to be in very good agreement with the experimental data prior to reshock by Latini, Schilling, and Don [Phys. Fluids 19, 024104 (2007)]. In the present investigation, the density, vorticity, baroclinic vorticity production, and simulated density Schlieren fields are first presented to qualitatively describe the reshock process. The baroclinic circulation deposition on the interface is shown to agree with the predictions of the Samtaney-Zabusky model and with linear instability theory. The time evolution of the positive and negative circulation on the interface is considered before and after reshock: it is shown that the magnitudes of the circulations are equal before as well as after reshock, until the interaction of the reflected rarefaction with the layer induces flow symmetry breaking and different evolutions of the magnitude of the positive and negative circulation. The post-reshock mixing layer growth is shown to be in generally good agreement with three models predicting linear growth for a short time following reshock. Next, a comprehensive investigation of local and global mixing properties as a function of time is performed. The distribution and amount of mixed fluid along the shock propagation direction is characterized using averaged mole fraction profiles, a fast kinetic reaction model, and mixing fractions. The modal distribution of energy in the mixing layer is quantified using the spectra of the fluctuating kinetic energy, fluctuating enstrophy, pressure variance, density variance, and baroclinic vorticity production variance. It is shown that a broad range of scales already exists prior to reshock, indicating that the single-mode Richtmyer-Meshkov instability develops nontrivial spectral content from its inception. The comparison of the spectra to the predictions of classical inertial subrange scalings in two-dimensional turbulence shows that the post-reshock spectra may be consistent with many of these scalings over wave number ranges less than a decade. At reshock, fluctuations in all fields (except for the density) are amplified across all scales. Reshock strongly amplifies the circulation, profiles, and mixing fractions, as well as the energy spectra and statistics, leading to enhanced mixing followed by a decay. The mole and mixing fraction profiles become nearly self-similar at late times following reshock; the mixing fraction exhibits an approach toward unity across the layer at the latest time, signifying nearly complete mixing of the gases. To directly quantify the amplification of fluctuations by reshock, the previously considered quantities are compared immediately after and before reshock. Finally, to investigate the decay of fluctuations in the absence of additional waves interacting with the mixing layer following reshock, the boundary condition at the end of the computational domain is changed from reflecting to outflow to allow the reflected rarefaction wave to exit the domain. It is demonstrated that the reflected rarefaction has an important role in breaking symmetry and achieving late-time statistical isotropy of the velocity field.
\end{abstract}

DOI: 10.1103/PhysRevE.76.026319

\section{INTRODUCTION}

The Richtmyer-Meshkov instability is a fundamental fluid instability that occurs when perturbations on an interface separating different gases grow following the passage of a

\footnotetext{
*Corresponding author. schilling1@1lnl.gov

†mlatini@acm.caltech.edu

*wsdon@cfm.brown.edu
}

PACS number(s): 47.20.Lz, 47.20.Ma, 47.20.Cq, 47.51.+a

shock. In the weighted essentially nonoscillatory (WENO) simulations of a model of the Mach 1.21 air(acetone)/SF 6 experiments of Collins and Jacobs [1] considered here (see Ref. [2] for details on the experiment and simulations), following the initial interaction at $0 \mathrm{~ms}$, a transmitted shock enters the second fluid. This shock reflects from the end wall of the shock tube test section and interacts with the evolving mixing layer at $\approx 6.4 \mathrm{~ms}$ in a process called reshock. Following reshock, a transmitted shock continues in the first fluid and a reflected rarefaction wave returns into the second fluid. 
The rarefaction wave reflects from the end wall and interacts again with the evolving interface at $\approx 11 \mathrm{~ms}$, generating a compression wave. The compression wave reflects from the end wall and again interacts with the evolving interface at $\approx 15 \mathrm{~ms}$. The growth and other mixing layer properties are affected by these wave-interface interactions, with each subsequent interaction further increasing fluctuations contributing to the mixing and formation of complex small-scale structures.

The physics of the reshocked Richtmyer-Meshkov instability is under intensive investigation due to its relevance to both core-collapse supernovae dynamics [3] and to inertial confinement fusion (ICF) [4-6]. The vast majority of simulations of ICF capsule implosions involving both the Rayleigh-Taylor and Richtmyer-Meshkov instabilities are still performed in one and two dimensions, as the computational resources required to adequately resolve such flows in three dimensions are still beyond the reach of available computing facilities. Thus ICF design will continue to rely on lower dimensional simulations, and it is therefore valuable to better understand the dynamics of these instabilities in two dimensions. In addition, it is of general interest to investigate theoretical aspects of two-dimensional turbulent flow induced by the Richtmyer-Meshkov instability. Moreover, very little previous investigation of the quantities considered in the present work has been performed in Richtmyer-Meshkov instability-induced mixing [7,8], particularly with reshock in either two or three dimensions. Here, data from ninth-order WENO simulations using a grid resolution of 512 points per initial perturbation wavelength $\lambda$ (the WENO9F simulation in Ref. [9]) is used to investigate the physics of reshock and mixing. In particular, the effects of reshock, as well as the decay properties at late times, are quantified. Both local and global quantities are considered. Two types of local quantities are considered at different times-profiles across the mixing layer and energy spectra. In the present geometry, mixing profiles are quantities averaged across the spanwise (y) direction perpendicular to the shock propagation direction (so that they are only a function of the streamwise coordinate $x$ and time) characterizing the distribution of mixing within the layer. Spectra of fluctuations within the mixing layer as a function of the wave number $k$ characterize the modal energy distribution in the mixing layer. Global quantities include mixing fractions and statistics as a function of time. Mixing fractions characterize the efficiency of mixing, including the effects of reshock and wave-interface interactions, and statistics characterize the evolution of energy as the mixing progresses. To investigate the effects of the reflected rarefaction wave on the mixing, simulations using an outflow and reflecting boundary condition were compared using data from the 256 points per initial perturbation wavelength simulation (the WENO9M simulation in Ref. [9]).

This paper is organized as follows. The equations solved and the suitability of the WENO method for investigating the physics of reshock and mixing are briefly discussed in Sec. II. The density, vorticity, baroclinic vorticity production, and simulated density Schlieren fields are used to visualize and qualitatively describe the reshock process in Sec. III. The circulation deposited on the interface by the shock is compared with the predictions of the Samtaney-Zabusky model and with linear instability theory in Sec. IV. The post-reshock mixing layer width is compared to the predictions of three phenomenological reshock models in Sec. V. The analysis of the local and global properties of mixing is presented in Sec. VI. Also presented is a comparison of mixing quantities and spectra immediately before and after reshock, and a comparison of results from reflecting and outflow boundary conditions at the end wall. A summary of the results and conclusions are given in Sec. VII.

\section{THE WENO METHOD FOR THE SIMULATION OF RESHOCK}

The reshocked Richtmyer-Meshkov instability must be simulated with some type of shock-capturing method. The fundamental issue with direct numerical simulation (DNS) is that it is well known that high-order (e.g., spectral or highorder finite-difference) schemes used for DNS are incapable of simulating shocked flows such as those arising from the Richtmyer-Meshkov instability due to Gibbs oscillations. In addition, it is not possible to fully resolve shocks in a macroscopic or continuum simulation in the sense required to consider it a DNS. Also, in simulations of reshocked Richtmyer-Meshkov instability using explicit molecular dissipation terms implemented in formally high-order shockcapturing schemes, the implicit dissipation exceeds the molecular dissipation upon shock passage (and especially upon reshock). Typically, implicit numerical diffusion present in the scheme or some type of subgrid-scale model (e.g., an "artificial viscosity") must be used, which is no more fundamental than any other methodology applied to this flow.

In the present simulations, the two-dimensional Euler equations augmented by the mass fraction conservation equation for the second gas,

$$
\frac{\partial \boldsymbol{\phi}}{\partial t}+\frac{\partial \boldsymbol{F}}{\partial x}+\frac{\partial \boldsymbol{G}}{\partial y}=0,
$$

are solved, where the conservative variables $\boldsymbol{\phi}$ and inviscid fluxes $\boldsymbol{F}$ and $\boldsymbol{G}$ are

$$
\begin{gathered}
\boldsymbol{\phi}=(\rho, \rho u, \rho v, \rho e, \rho m)^{T}, \\
\boldsymbol{F}=\left(\rho u, \rho u^{2}+p, \rho u v,(\rho e+p) u, \rho m u\right)^{T}, \\
\boldsymbol{G}=\left(\rho v, \rho u v, \rho v^{2}+p,(\rho e+p) v, \rho m v\right)^{T} .
\end{gathered}
$$

Here, $\rho$ is the density, $\mathbf{u}=(u, v)$ is the velocity, $p$ is the pressure, $e=\left(u^{2}+v^{2}\right) / 2+p /[\rho(\gamma-1)]$ is the total energy per unit mass, $m$ is the mass fraction (here of the denser sulfur hexafluoride gas, $\mathrm{SF}_{6}$ ), and $p=\rho R T$ is the ideal gas pressure ( $R$ is the gas constant).

The simulations were performed using the finitedifference WENO shock-capturing method [10]. A single gamma is used in the present simulations as multiple gammas create nonphysical pressure oscillations near the material interfaces (which are nontrivial to mitigate) [11-14]. Although the transmitted and reflected shock speeds, time of reshock, and initial interface velocity would change in a twogamma formulation [2], the general trends in the mixing 


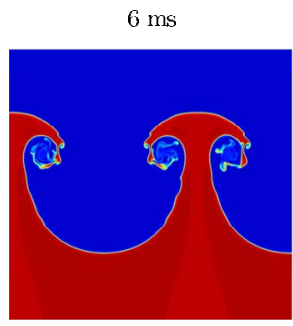

$6.68 \mathrm{~ms}$
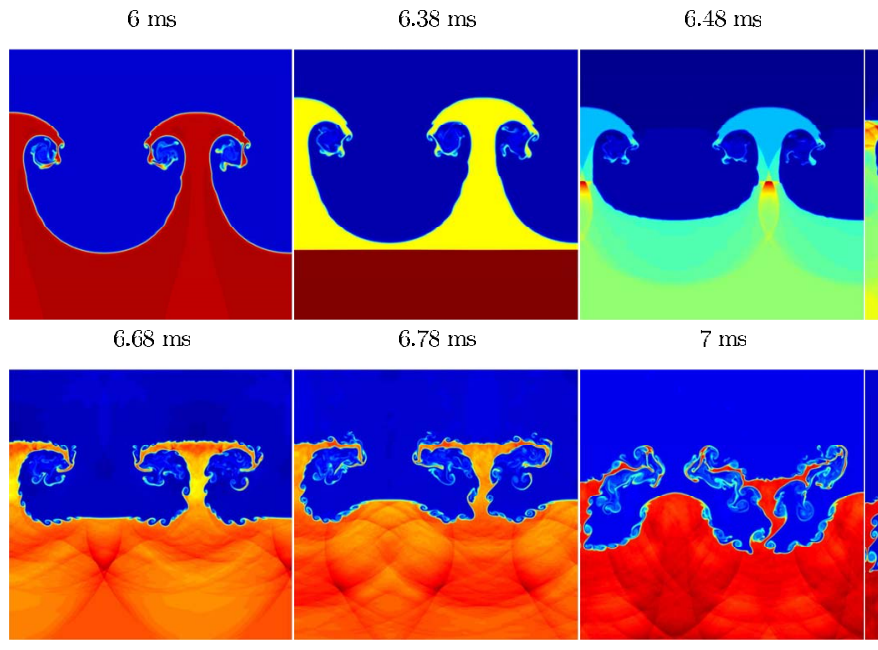

$7 \mathrm{~ms}$
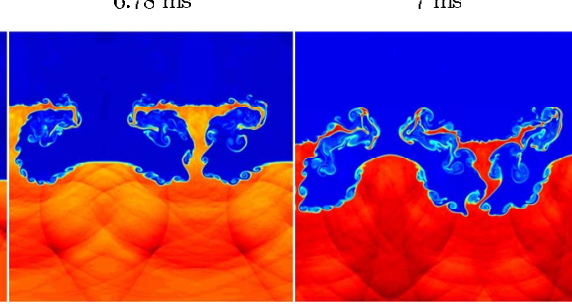

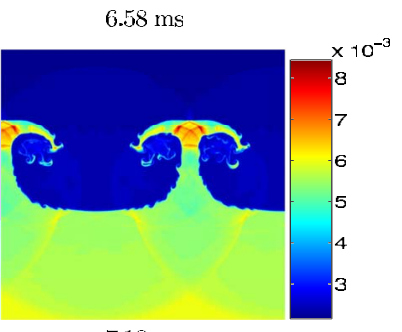

$7.18 \mathrm{~ms}$

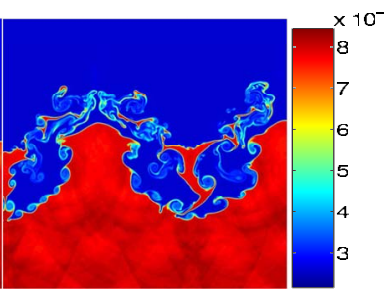

FIG. 1. (Color online) The density fields (in units of $\mathrm{g} / \mathrm{cm}^{3}$ ) at $6,6.38,6.48,6.58,6.58,6.68$, $6.78,7$, and $7.18 \mathrm{~ms}$. The gases are air (acetone) (top) and $\mathrm{SF}_{6}$ (bottom). properties and the overall conclusions of the present study are not expected to change significantly, as the flow becomes nearly incompressible, following shock passage $[15,16]$. The conservative finite-difference discretization of the Euler equations with WENO flux reconstruction contains implicit truncation errors that can be regarded as a nonlinear, adaptive numerical dissipation. Although the Euler equations are solved in the present work, it is possible to compare the kinematic viscosity of the mixture, $\nu_{\text {mix }}$, in the Collins and Jacobs experiment and the implicit numerical viscosity $\nu_{\text {num }}$. The kinematic viscosities of air (acetone) and $\mathrm{SF}_{6}$ are 0.156 and $0.026 \mathrm{~cm}^{2} / \mathrm{s}$, respectively [17]. Estimating the mixture dynamic viscosity by [18]

$$
\mu_{\mathrm{mix}}=\frac{X_{1} \mu_{1} \sqrt{M_{1}}+X_{2} \mu_{2} \sqrt{M_{2}}}{X_{1} \sqrt{M_{1}}+X_{2} \sqrt{M_{2}}}
$$

where $X_{r}$ are the mole fractions of the mixture components and $M_{r}$ are the molecular weights [2], gives $\nu_{\text {mix }}$ $=\mu_{\text {mix }} / \rho_{\text {mix }}=0.047 \mathrm{~cm}^{2} / \mathrm{s}$, where $\rho_{\text {mix }}=\left(\rho_{1}+\rho_{2}\right) / 2$. The computed numerical viscosity $\nu_{\text {num }} \approx 0.1 \mathrm{~cm}^{2} / \mathrm{s}$ following reshock [9] is close to the estimated $\nu_{\text {mix }}$. In a previous study [9], the effects of order of WENO flux reconstruction and grid resolution on the quantities considered here showed that these quantities exhibited little sensitivity to order and resolution prior to reshock. However, following reshock, higher order methods preserved more small-scale structure and had lower numerical dissipation than lower-order methods on the same grid. Furthermore, in Ref. [2] the agreement between the WENO densities and the experimental planar laserinduced fluorescence (PLIF) images, as well as the agreement of the amplitude with the experimental data and with the predictions of linear, semianalytical, and empirical models, provided an element of validation of this numerical method. These results support using the ninth-order WENO method in investigating shock-interface interactions, including the reshocked Richtmyer-Meshkov instability.

\section{THE PHENOMENOLOGY OF RESHOCK}

A temporal sequence of the density, vorticity, baroclinic vorticity production, and simulated density Schlieren fields is used here to qualitatively describe the phenomenology of reshock, including the effects of vorticity deposition on the interface, shock focusing, and the effects of reflected waves on the interface evolution. Images of the vortex rollup are also considered to describe the internal structure of the core during reshock.

\section{A. The density fields}

Reshock is first visualized through the time sequence of density fields shown in Fig. 1 from 6 to $7.18 \mathrm{~ms}$. At $6 \mathrm{~ms}$ (prior to reshock), the densities exhibit small-scale structure inside the rollup, which forms as a result of the secondary vorticity deposition from the interface rollup [19]. Reshock is a refraction from a heavier gas $\left(\mathrm{SF}_{6}\right)$ into a lighter gas (air and acetone mixture) [air (acetone) in the sequel], resulting in a transmitted shock that enters the air (acetone) and a reflected rarefaction that returns back into the $\mathrm{SF}_{6}$. The densities show the arrival of the reflected shock at $6.38 \mathrm{~ms}$, the shock refraction and the transmitted shock in air (acetone), the reflected rarefaction returning into the $\mathrm{SF}_{6}$ at 6.48 and $6.58 \mathrm{~ms}$, the inversion process when the bubble transforms into a spike (and vice versa) at 6.58, 6.68, and $6.78 \mathrm{~ms}$, and the formation of additional small rollups and complex disordered structures at 7 and $7.18 \mathrm{~ms}$. Curved waves can be seen in the $\mathrm{SF}_{6}$ at $6.58,6.68$, and $6.78 \mathrm{~ms}$. At late times $(11,15$, and $18 \mathrm{~ms}$ ) shown in Fig. 2, a large spike of $\mathrm{SF}_{6}$ develops. A finely mixed layer is observed, which is the remnant of the spike prior to reshock. The creation of small disordered structures breaks symmetry and forms a complex interface topology. This is also qualitatively observed in the experimental density images [1].

\section{B. The vorticity and baroclinic vorticity production fields}

The vorticity evolution equation (in two dimensions and in the absence of dissipation) is

$$
\frac{d \omega}{d t}=\mathcal{P}+\mathcal{C}, \quad \mathcal{P} \equiv \frac{\nabla \rho \times \nabla p}{\rho^{2}}, \quad \mathcal{C} \equiv-\boldsymbol{\omega} \nabla \cdot \boldsymbol{u},
$$

where $d / d t=\partial / \partial t+\nabla \cdot \mathbf{u}, \mathcal{P}$ is the baroclinic vorticity production term, and $\mathcal{C}$ is the compression term; $\omega$ and $\mathcal{P}$ are shown 
$11 \mathrm{~ms}$

$15 \mathrm{~ms}$

$18 \mathrm{~ms}$

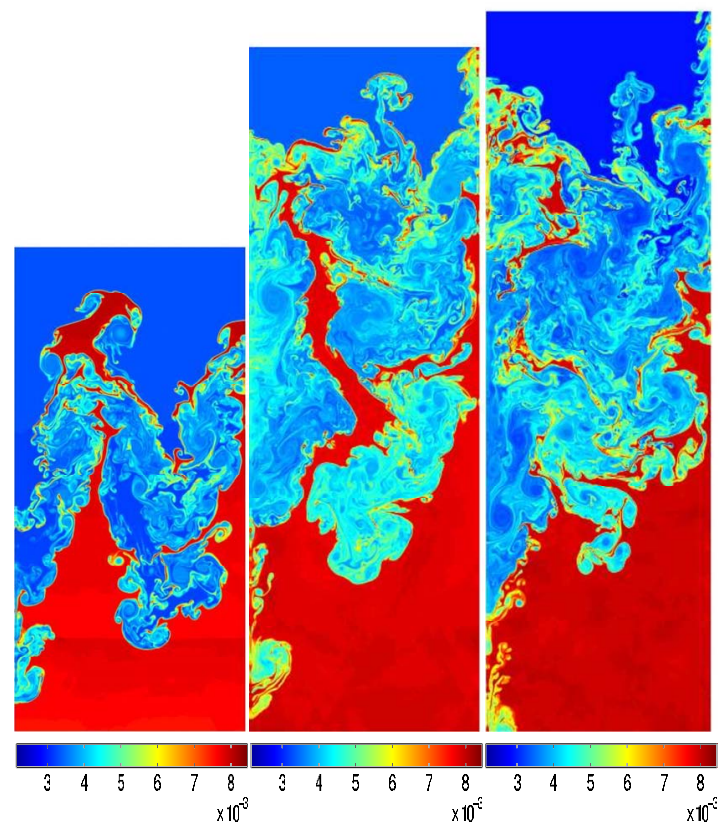

FIG. 2. (Color online) Same as Fig. 1 but at 11, 15, and $18 \mathrm{~ms}$.

in Fig. 3 at the same times as the density fields in Fig. 1. Baroclinic vorticity production causes vorticity deposition with opposite sign on the interface during reshock: this term is approximately two orders of magnitude larger than prior to reshock. The vorticity rolls up into strong cores prior to reshock at 6 and $6.38 \mathrm{~ms}$. The density and pressure fluctuations along the interface cause the deposition of additional vorticity, as seen in $\mathcal{P}$ at the corresponding times. During reshock at $6.48 \mathrm{~ms}$, the baroclinic vorticity production is strongly increased along the interface, particularly on the sides of the bubbles, where the density and pressure gradient misalignment is largest. The vorticity deposited is also approximately four times larger than the vorticity in the spike cores prior to reshock. The vorticity of opposite sign subsequently drives the inversion process, which transforms the bubbles into spikes and vice versa, and induces the formation of additional disordered complex structures at 7 and $7.18 \mathrm{~ms}$. Following shock passage, baroclinic production becomes much smaller.

The vorticity and baroclinic vorticity production fields are shown in Fig. 4 at late times following reshock (11, 15, and $18 \mathrm{~ms}$ ). The vorticity fragments into small localized vortices at late times. Baroclinic production is active between the two interfaces and contributes to the development of complex structures and to increased mixing. At late times, $\mathcal{P}$ is approximately two orders of magnitude smaller than at reshock. Furthermore, it remains approximately constant at late times, indicating that the arrival of the reflected rarefaction at $\approx 11 \mathrm{~ms}$ and of the reflected compression at $\approx 15 \mathrm{~ms}$ does not significantly affect baroclinic production.

A closeup of the density, vorticity, and baroclinic vorticity production fields inside the rollup is shown in Fig. 5 during and after reshock. The density shows the formation of complex structures, the vorticity shows the dynamical mecha- nisms driving the formation of these structures, and the baroclinic vorticity production shows the mechanisms driving the vorticity through the misalignment of $\nabla \rho$ and $\nabla p$ inside the vortex core as the rollup progresses. The baroclinic vorticity production increases at $6.58 \mathrm{~ms}$ in the rollup, corresponding to the passage of one of the transmitted shocks during reshock. This shock deposits vorticity of opposite sign on the interface, which drives the inversion process and the formation of additional complex small-scale structures.

\section{The simulated density Schlieren fields}

The detailed wave structure can be investigated using simulated density Schlieren fields, which visualize density gradients associated with the waves and fine-scale mixing structures. The density Schlieren is that used in twodimensional fifth-order WENO simulations of a shockcylinder interaction [20],

$$
\Phi(x, y, t)=\exp \left[-\alpha(m) \frac{|\nabla \rho|}{\max |\boldsymbol{\nabla} \rho|}\right],
$$

where $\alpha(m)=20$ for $m>0.25$ and 100 otherwise, and $m$ is the mass fraction of $\mathrm{SF}_{6}$.

A temporal sequence of simulated density Schlieren fields during reshock and at late times is shown in Fig. 6. The fields sharply capture the diffuse interface, the complex wave structure during reshock, and the small-scale structures at late times. Note the focusing effect of the waves and the bubble transforming into the spike and vice versa. Reshock occurs over a time scale of $\approx 0.2-0.3 \mathrm{~ms}$. The field at $11 \mathrm{~ms}$ shows the arrival of the reflected rarefaction produced by the second shock after it has reflected from the end wall of the domain. The rich wave structure displayed by $\Phi$ shows weak, curved waves that intersect and cause focusing. As these waves are weak due to the small Mach number, it is unclear whether they have an important role in the flow dynamics. At late times, the waves are no longer present in the unmixed fluids. Similar complex wave structures were also observed following a shock-interface interaction in the Mach 1.32 experimental Schlieren images of Brouillette and Sturtevant $[21]$.

\section{BAROCLINIC CIRCULATION DEPOSITION ON THE INTERFACE}

The circulation deposited on the interface by the shock quantifies the principal mechanism driving the RichtmyerMeshkov instability evolution. Reshock imparts additional circulation on the interface, contributing to the rapid development of complex flow structure through baroclinic production.

Consider a closed oriented curve $C$ enclosing a surface $S$, so that the circulation is

$$
\Gamma(t)=\oint_{C} \boldsymbol{u} \cdot d \boldsymbol{r}=\int_{S} \boldsymbol{\omega} \cdot d \boldsymbol{S},
$$




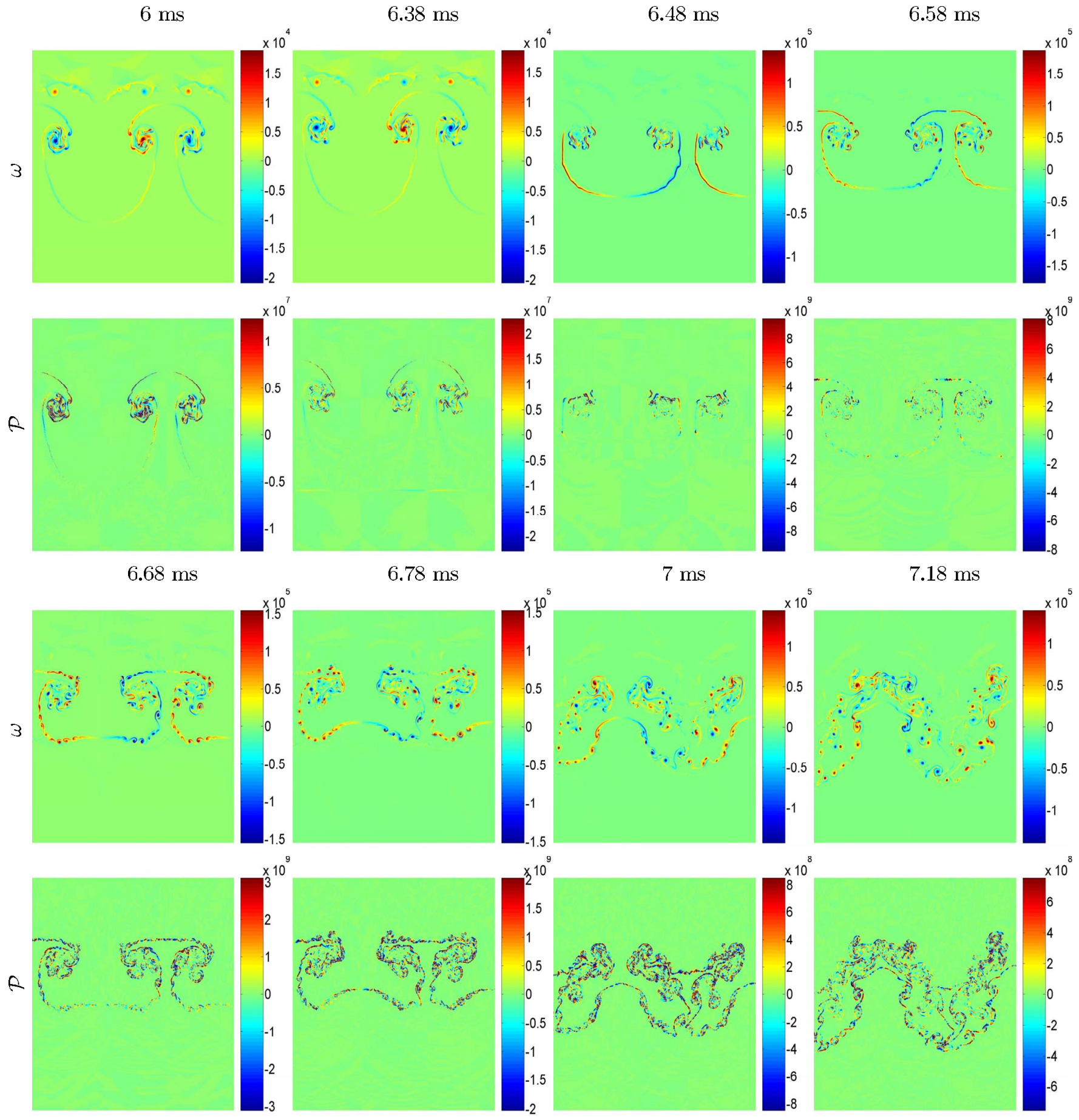

FIG. 3. (Color online) The vorticity $\omega$ (in units of $\mathrm{s}^{-1}$ ) and baroclinic vorticity production $\mathcal{P}$ (in units of $\mathrm{s}^{-2}$ ) fields at $6,6.38,6.48,6.58$, $6.68,6.78,7$, and $7.18 \mathrm{~ms}$.

where $d \boldsymbol{r}$ is tangent to $C$ and $d \boldsymbol{S}$ is the outward oriented area element. Here, the vorticity vector and the area element are parallel; the latter definition for computing the circulation is adopted, with $d \boldsymbol{S}$ taken to be a rectangle. In the limit of a rectangle with infinite length in the streamwise $(x)$ direction and negligible width in the spanwise $(y)$ direction, the circulation on the interface is approximated as $\langle\omega\rangle(y, t)$ $\equiv \int_{-\infty}^{\infty} \omega(x, y, t) d x$.
Samtaney and Zabusky [22] analytically derived

$$
\langle\omega\rangle\left(y, 0^{+}\right)=\Gamma_{1}^{\prime} k a_{0}^{+} \sin (k y)
$$

for the circulation deposition on a sinusoidal interface by the initial incident shock in a fast or slow interaction (i.e., when the refraction is from a lighter fluid into a heavier fluid), where 
$11 \mathrm{~ms}$
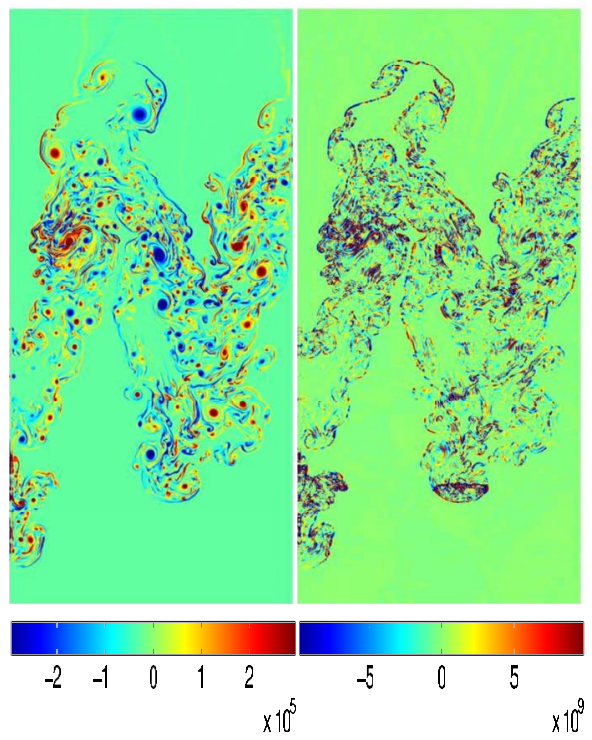

$\omega$
$\mathcal{P}$
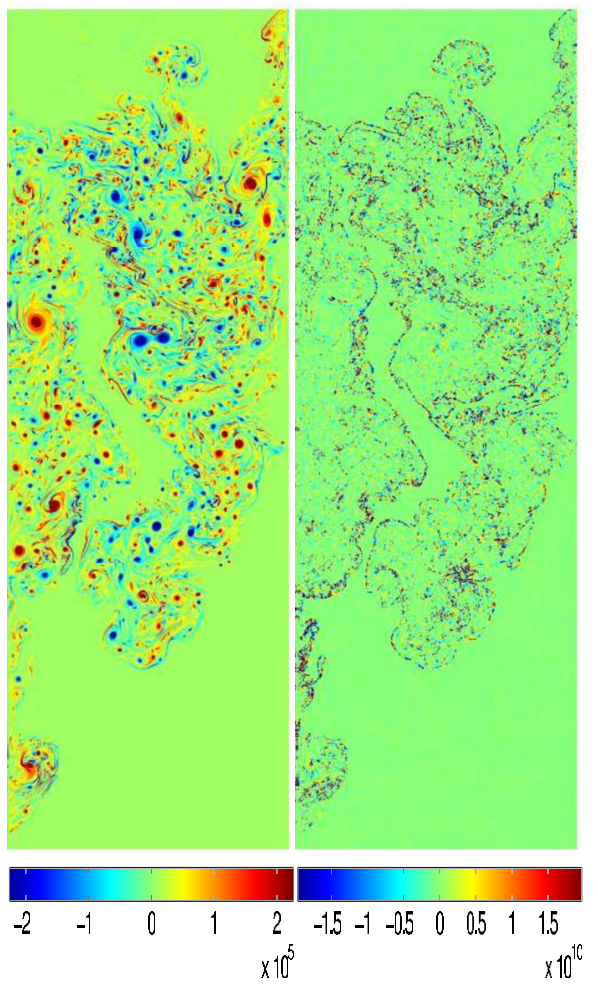

$\omega$
$P$
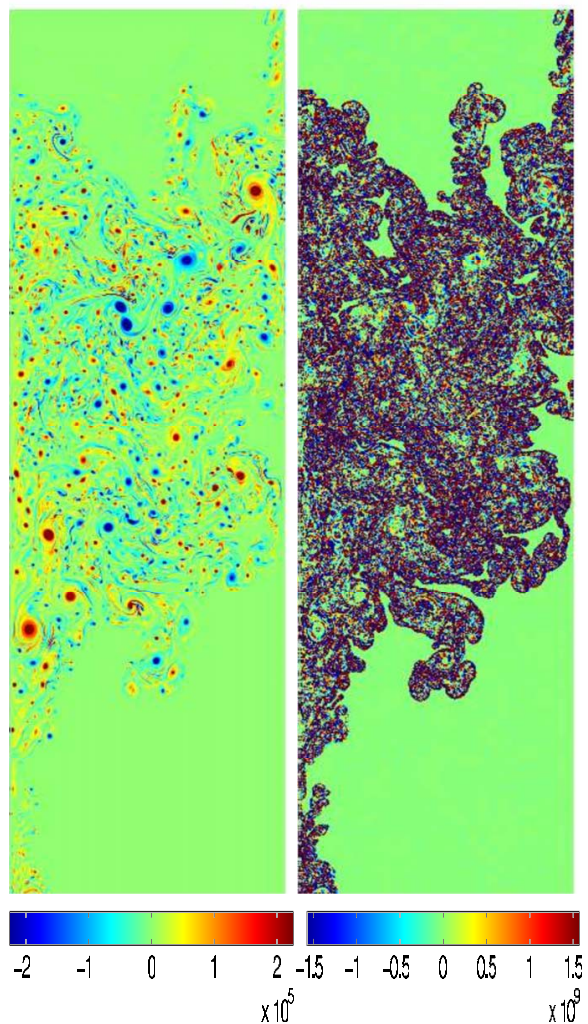

$\mathcal{P}$

FIG. 4. (Color online) Same as Fig. 3 but at 11, 15, and $18 \mathrm{~ms}$.

$$
\Gamma_{1}^{\prime}=\frac{c_{1}}{\mathrm{Ma}}\left(\frac{1}{\gamma_{2}-1} \frac{1-\psi\left(p_{4} / p_{2}\right)}{\eta \gamma_{1} / \gamma_{2}}-\frac{1-\psi\left(p_{5} / p_{3}\right) \psi\left(p_{3} / p_{1}\right)}{\gamma_{1}-1}\right)
$$

$\mathrm{Ma}=1.21$ is the incident shock Mach number, $p_{1}=p_{2}$ is the initial pressure ahead of the incident shock, $p_{3}$ is the pressure behind the incident shock, $p_{4}=p_{5}$ is the pressure behind the reflected and transmitted shocks, $\eta$ is the initial density ratio, $c_{1}$ is the initial sound speed, the ratio of sound speed across the incident, reflected and transmitted shocks is given by $\sqrt{\psi\left(p_{3} / p_{1}\right)}, \sqrt{\psi\left(p_{5} / p_{3}\right)}$, and $\sqrt{\psi\left(p_{4} / p_{2}\right)}$, respectively, $\psi(r)$ $\equiv r(1+\mu r) /(\mu+r)$, and $\mu \equiv(\gamma+1) /(\gamma-1)$ (where $\gamma=1.276)$. In the present simulation, $\Gamma_{1}^{\prime}=8736.12 \mathrm{~cm} / \mathrm{s}$, the perturbation wave number is $k=2 \pi / \lambda=1.065 \mathrm{~cm}^{-1}$, and the postshock perturbation amplitude is $a_{0}^{+}=0.162 \mathrm{~cm}$. The initial interfacial circulation deposited by the incident shock, together with the prediction of Eq. (8), are shown in Fig. 7, indicating that the circulation is sinusoidally distributed and closely follows the Samtaney-Zabusky model prediction. The model underpredicts the simulation data by $\approx 4 \%$. Also shown is a comparison with linear instability theory $\left\langle\omega\left(y, 0^{+}\right)\right\rangle=d \mu / d y$, where $\mu(y)=2 v_{0} \cos (k y)$ and $v_{0}=702 \mathrm{~cm} / \mathrm{s}$ is the initial instability growth rate, indicating that linear theory is in close agreement with the Samtaney-Zabusky model and also underpredicts the simulation data.
As the circulation deposition is sinusoidal, the regions $S^{-}=\left[0, L_{x}\right] \times[0, \lambda / 2]$ and $S^{+}=\left[0, L_{x}\right] \times[\lambda / 2, \lambda]$ enclose the circulation of the same sign. Thus define the positive and negative circulations

$$
\Gamma^{ \pm}(t) \equiv \iint_{S^{ \pm}} \omega(x, y, t) d x d y
$$

(see [9] for details on their numerical computation) which, as shown in Fig. 8, overlap prior to reshock, indicating symmetry as the instability evolves. At $\approx 6 \mathrm{~ms}$, a small decrease resulting from the deposition of vorticity of opposite sign [19] is observed (this can also be seen in the rollup in Fig. 5, where the vorticity forms positive and negative patches). Reshock at $\approx 6.4 \mathrm{~ms}$ deposits vorticity of opposite sign on the interface, as the refraction is from a heavier gas into a lighter gas. Consequently, both circulations increase sharply and change sign. The large values of $\left|\Gamma^{ \pm}\right|$following reshock are due to the more complex topology of the interface, resulting in a larger amount of vorticity deposited by the shock. The secondary baroclinic circulation also explains the more pronounced increase in $\left|\Gamma^{ \pm}\right|$following reshock, but prior to the arrival of the reflected rarefaction. Following the arrival of the reflected rarefaction, $\left|\Gamma^{+}\right|$and $\left|\Gamma^{-}\right|$begin to differ as a result of symmetry breaking. Vortical structures become disordered and may cross from the positive region into the 

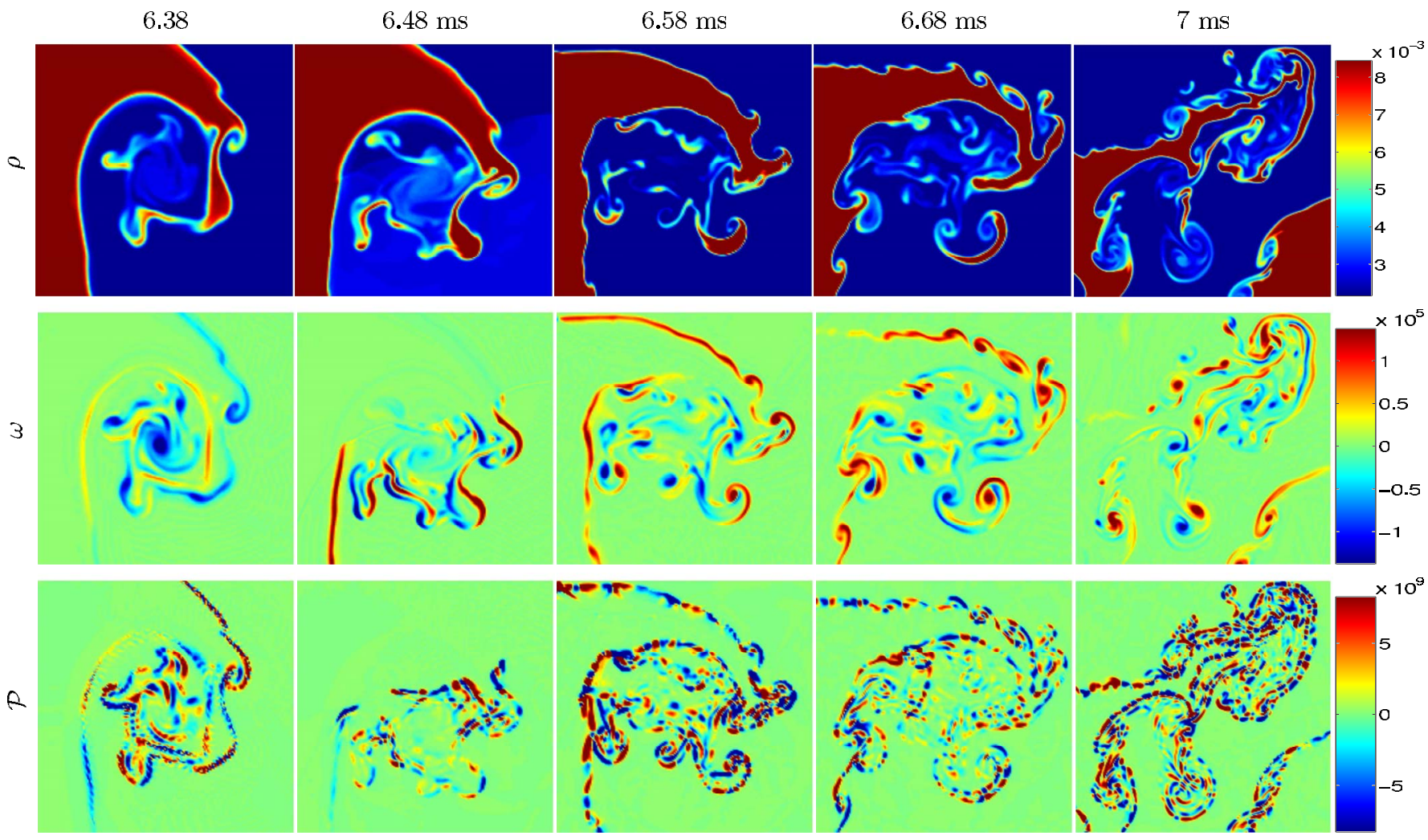

FIG. 5. (Color online) Details of the rollup in the density $\rho$, vorticity $\omega$, and baroclinic vorticity production $\mathcal{P}$ fields at $6.48,6.58,6.68$, and $7 \mathrm{~ms}$. The images show the complex structure in the rollup, including the effects of reshock on this structure.

negative region, inducing the asymmetry. Baroclinic secondary instability and the rapid growth of positive and negative circulation was investigated in a two-dimensional stratified mixing layer [23-25].

\section{THE POST-RESHOCK MIXING LAYER WIDTH}

The post-reshock mixing layer width is compared here to the phenomenological Mikaelian [26], Brouillette and Sturtevant [27], and Charakhch'yan [28] reshock models, which predict linear growth for short times following reshock. These models apply up to the time of the interaction with the reflected rarefaction at $\approx 11 \mathrm{~ms}$. The numerical determination of the mixing layer width is discussed in detail in Ref. [2].

\section{A. The Mikaelian reshock mode}

Mikaelian [26] derived a linear power-law model for the post-reshock mixing layer width based on the rocket-rig experimental results of Read [29] and Youngs [30] for the growth of the Rayleigh-Taylor unstable mixing layer. Assuming no dependence on the initial conditions and neglecting molecular dissipation (and other) effects [31],

$$
\frac{d h}{d t}=0.28 \Delta u_{1} A_{1}^{+}
$$

where $\Delta u_{1}$ is the change in the interface velocity resulting from reshock, $A_{1}^{+}$is the post-reshock Atwood number, and the empirical coefficient 0.28 is based on the mixing layer growth rate from the experiments [29].

\section{B. The Brouillette-Sturtevant reshock model}

Brouillette and Sturtevant [27] studied the effect of a diffuse interface on Richtmyer-Meshkov instability growth in shock tube experiments in which a thin metal plate initially separated air and $\mathrm{SF}_{6}$ or air and Freon-22. Prior to launching the shock in the air, the plate was slowly withdrawn to generate a quasisinusoidal perturbation, leaving a thick diffuse interface separating the gases. The Mach 1.12-1.66 shock refracted at the interface and was transmitted into the second gas. The transmitted shock reflected from the end wall of the shock tube test section and reshocked the interface.

The growth of the total width of a single-mode interface following $N+1$ impulsive accelerations was empirically modeled by generalizing the Richtmyer model:

$$
\left(\frac{d h}{d t}\right)_{N}=2 k \sum_{i=0}^{N} \frac{\Delta u_{i} A_{i}^{+} a_{i}^{+}}{\psi_{i}^{+}},
$$

where $k$ is the initial perturbation wave number, $\Delta u_{i}$ is the change in the velocity of the interface corresponding to the $i$ th wave, $A_{i}^{+}$and $a_{i}^{+}$are the post-(re)shock Atwood number and amplitude, $\psi_{i}^{+}$is the post-shock growth reduction factor [32], and the factor of 2 accounts for the width (twice the amplitude $a$ ). As the growth is based on the Richtmyer model for each impulsive acceleration phase, the BrouilletteSturtevant model predicts piecewise-linear-in-time amplitude 
$6.38 \mathrm{~ms}$

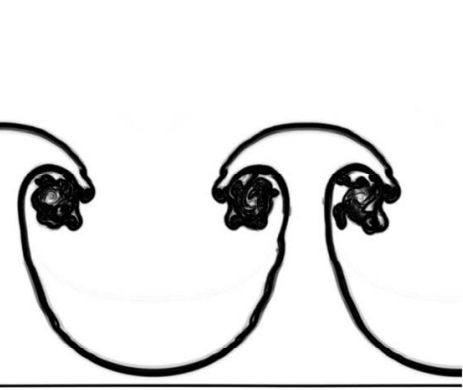

$6.78 \mathrm{~ms}$
$6.48 \mathrm{~ms}$

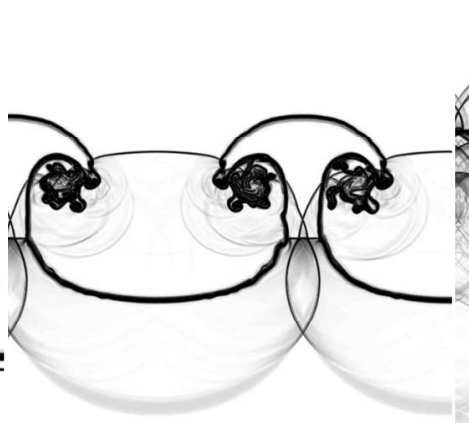

$6.58 \mathrm{~ms}$

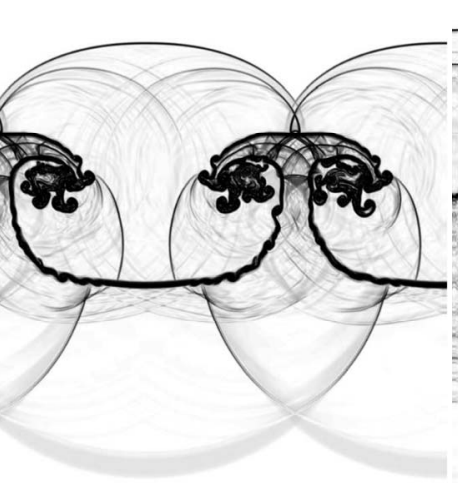

$11 \mathrm{~ms}$
$6.68 \mathrm{~ms}$

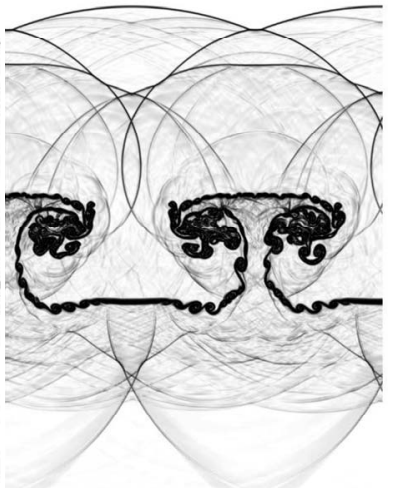

$7.18 \mathrm{~ms}$

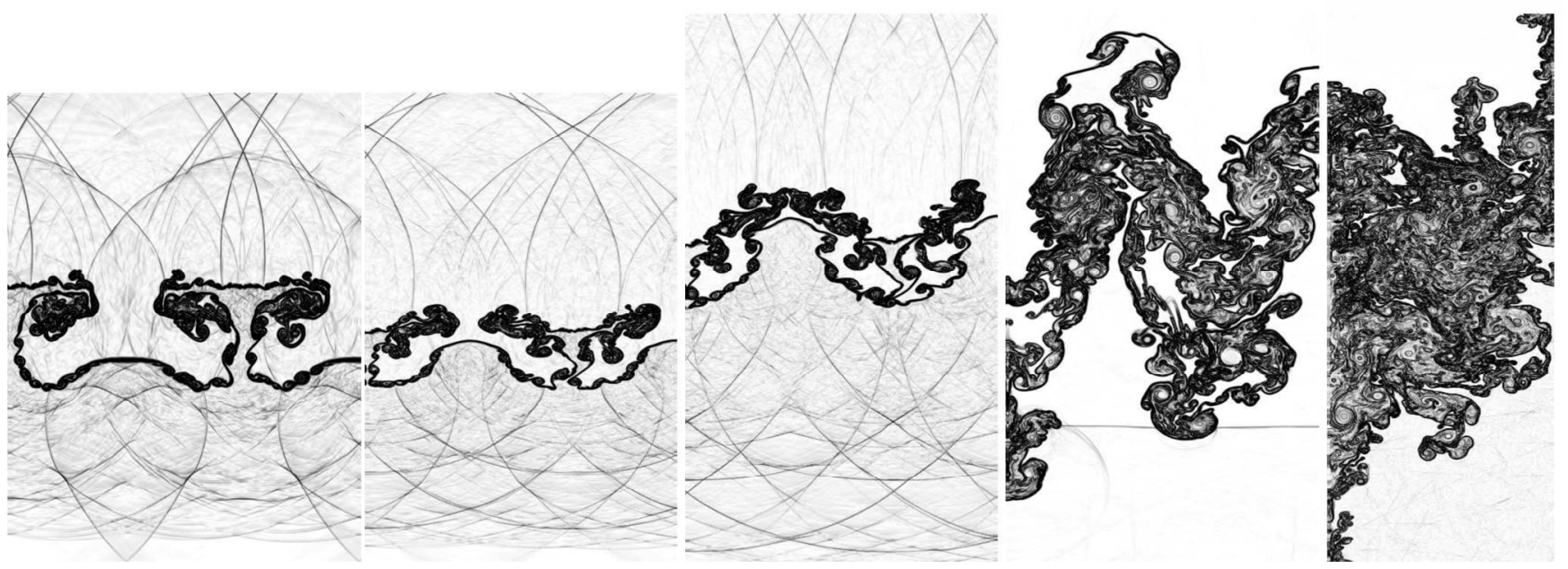

$18 \mathrm{~ms}$

FIG. 6. Simulated density Schlieren fields $\Phi$ showing the complex wave structure during reshock, shock focusing, and the fine-scale structure of the interface at late times.

growth phases. This model also accounts for the multiple waves that successively interact with the interface. Brouillette and Sturtevant reported good agreement between their data and Eq. (12).

\section{The Charakhch'yan reshock model}

Charakhch'yan [28] assumed that the reshock is much weaker than the initial incident shock and occurs during the nonlinear phase when the amplitude is $a(t) \sim \lambda$. As a result, the change in Atwood number caused by reshock can be neglected and

$$
\frac{d h}{d t}=2 v_{0}-2.5 \Delta u_{1} A^{+}
$$

where $\mathrm{A}^{+}$is the Atwood number following the passage of the initial shock, the empirical coefficient 2.5 is based on twodimensional simulations, and $d a_{r}^{-} / d t$ in Ref. [28] was interpreted as the Richtmyer velocity $v_{0}$. A wide range of numerical simulations was reported to support this model.

\section{Comparison of the post-reshock mixing layer width to model predictions}

The comparison of the post-reshock mixing layer width to the model predictions is shown in Fig. 9. Reshock in the simulation occurs at nearly the same time as in the experiment, as confirmed by both the $x$ - $t$ diagram and the mixing layer amplitude in Ref. [2]. For the Mikaelian model, the simulation values $\Delta u_{1}=8000 \mathrm{~cm} / \mathrm{s}$ and $A_{1}^{+}=0.6448$ are used. For the Brouillette-Sturtevant model,

$$
\left(\frac{d h}{d t}\right)_{1}=2 k\left(\frac{\Delta u A^{+} a_{0}^{+}}{\psi^{+}}+\frac{\Delta u_{1} A_{1}^{+} a_{1}^{+}}{\psi_{1}^{+}}\right)
$$

was used, where the post-reshock amplitude is $a_{1}^{+}=1.5 \mathrm{~cm}$, $\Delta u_{1}=8000 \mathrm{~cm} / \mathrm{s}$, and the value of $\psi_{1}^{+}$was extrapolated as follows. First, recall that $\psi$ is linear in $\delta / \lambda$, where $\delta$ is the diffuse mixing layer width (linear in the post-shock perturbation amplitude $a_{0}^{+}$). As $a_{1}^{+} \approx 10 a_{0}^{+}$and the value of $\psi^{+}$is known, the new value of $a_{1}^{+}$gives the new value of $\psi_{1}^{+}$. The value $A^{+}=0.6045$ from Table I of Ref. [2] was used for the Charakhch'yan model.

Agreement between the mixing layer width and the model predictions can only be expected following reshock for $t$ $>6.4 \mathrm{~ms}$ and prior to the arrival of the reflected rarefaction 


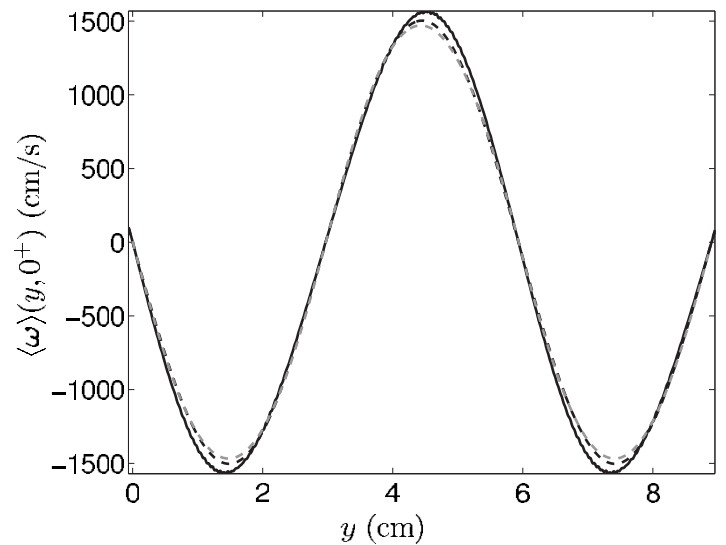

FIG. 7. The initial circulation deposition on the interface $\langle\boldsymbol{\omega}\rangle\left(y, 0^{+}\right)$(solid line) by the incident shock at time $t=0^{+}$, together with the predictions of the Samtaney-Zabusky [Eq. (8)] model (dashed line) and linear theory (dashed gray line).

at $\approx 11 \mathrm{~ms}$. Comparison of $h(t)$ with the model predictions indicates that the growth is approximately linear for very short times $(<2 \mathrm{~ms})$ following reshock. The simulation width generally agrees well with the both the Mikaelian and Charakhch'yan model predictions at early times following reshock. Vetter and Sturtevant [31] also concluded that the widths from their reshocked air/SF 6 experiments agreed with the Mikaelian model within 1.5-23\%, depending on the shock strength. For later times, the Mikaelian and
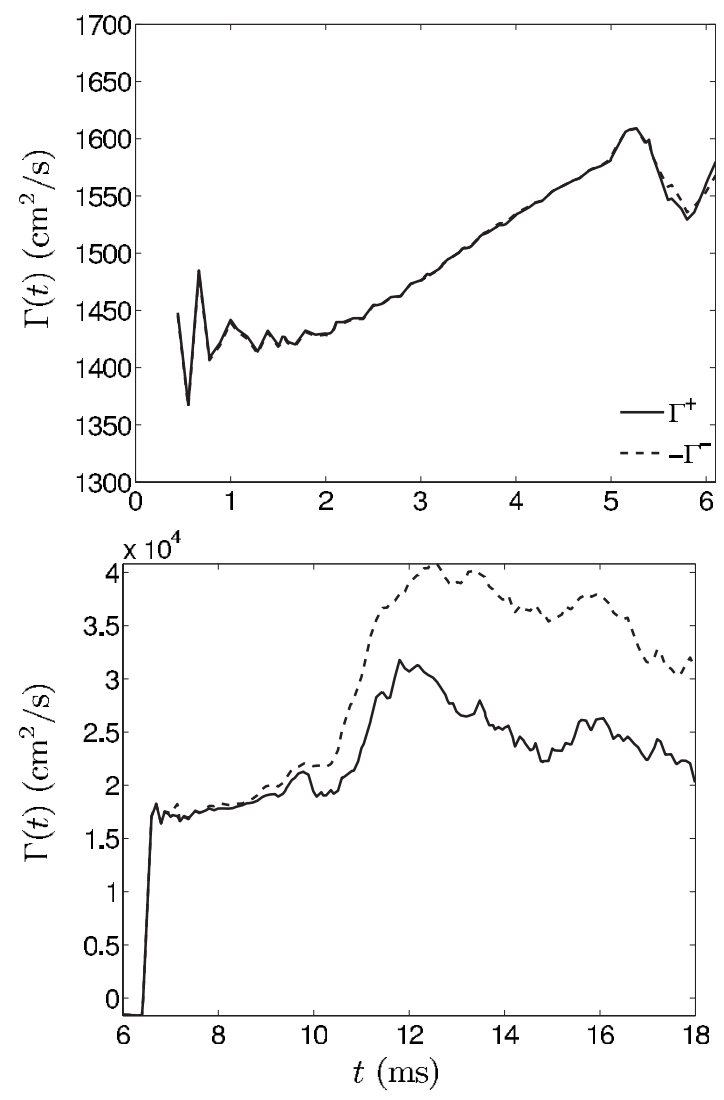

FIG. 8. The time evolution of the positive and negative circulation $\Gamma^{ \pm}(t)$ on the interface before (top) and after (bottom) reshock.

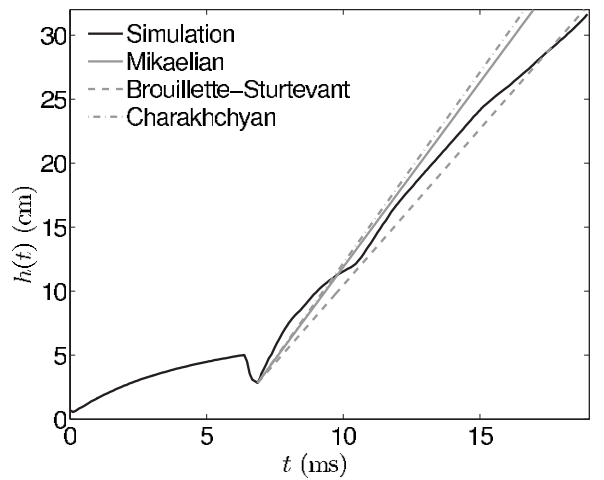

FIG. 9. The mixing layer width $h(t)$ showing reshock at $\approx 6.4 \mathrm{~ms}$ and the arrival of the reflected rarefaction at $\approx 11 \mathrm{~ms}$. Also shown are the predictions of the Mikaelian, Brouillette-Sturtevant, and Charakhch'yan models. The model predictions are displayed starting at $t_{r}^{+}=6.58 \mathrm{~ms}$ and at $h\left(t_{r}^{+}\right)=3.1 \mathrm{~cm}$. Using the values in the text yields $(d h / d t)_{1}=1444.35 \mathrm{~cm} / \mathrm{s},(d h / d t)_{1}=1195.1 \mathrm{~cm} / \mathrm{s}$, and $(d h / d t)_{1}=1492 \mathrm{~cm} / \mathrm{s}$ for the Mikaelian, Brouillette-Sturtevant, and Charakhch'yan models, respectively. The variations in the growth rate at $\approx 11$ and $15 \mathrm{~ms}$ are due to the further wave interactions with the evolving interface, and are not accounted for by these models.

Charakhch'yan models overestimate the growth, while the Brouillette-Sturtevant model underestimates the growth at all times following reshock. These reshocked amplitude growth predictions only depend on the model parameters $\Delta u_{1}$ and $A_{1}^{+}$. The Mikaelian and Charakhch'yan models do not depend on $k$ (in contrast to the Brouillette-Sturtevant model) and contain empirical parameters. The Mikaelian model is based on an estimate of the growth rate of a three-dimensional multimode Rayleigh-Taylor experiment, while the Brouillette-Sturtevant and Charakhch'yan models are fundamentally two-dimensional single-mode (and thus more consistent with the present simulations).

\section{ANALYSIS OF MIXING AND THE PHYSICS OF RESHOCK}

Presented here is an investigation of quantities characterizing the evolution of mixing induced by the reshocked single-mode Richtmyer-Meshkov instability. The quantities are adapted from similar investigations of the RayleighTaylor instability [33] and, more generally, of turbulent flows. The variety of quantities considered allows a quantitative estimate of the effects of reshock. The effects of subsequent wave-interface interactions on the evolution of the layer are also considered.

The initial condition is deterministic in the present study. Furthermore, only one realization of the flow is simulated. Thus the profiles, spectra, and statistics exhibit variations that would otherwise be reduced if an ensemble average of realizations with stochastic initial conditions was used. The observed trends, however, are not expected to differ significantly between a single realization and an ensemble average. 


\section{A. Definitions of averages, fluctuations, spectra, and statistics}

Several averages are introduced to define streamwise profiles across the mixing layer, as well as fluctuations required to define energy spectra. Let an overbar denote an instantaneous average of a field $\phi(x, y, t)$ over the spanwise direction $y$ with length $L_{y}$ :

$$
\bar{\phi}(x, t)=\frac{1}{L_{y}} \int_{0}^{L_{y}} \phi(x, y, t) d y, \quad \widetilde{\phi}(x, t)=\frac{\overline{\rho \phi}}{\bar{\rho}},
$$

which can be interpreted as an instantaneous Reynolds average (see Ref. [7] for the three-dimensional analog) and an instantaneous Favre average, respectively; the latter is used for the statistical analysis of variable-density and compressible flows $[34,35]$. Then the corresponding Reynolds and Favre fluctuating fields are

$$
\begin{aligned}
& \phi(x, y, t)^{\prime}=\phi(x, y, t)-\bar{\phi}(x, t), \\
& \phi(x, y, t)^{\prime \prime}=\phi(x, y, t)-\widetilde{\phi}(x, t) .
\end{aligned}
$$

Thus, in the present analysis, the Reynolds- and Favreaveraged fields are functions only of the streamwise coordinate $x$ and of time, while the fluctuating fields are functions of both the streamwise and spanwise coordinates $x$ and $y$, and of time.

The time evolution of the fluctuating kinetic energy, fluctuating enstrophy, density variance, pressure variance, and baroclinic vorticity production variance spectra quantify the growth of different scales of the flow, both before and after reshock. The spectral analysis used here adapts the formulations previously applied to inhomogeneous flows with one assumed direction of (approximate) statistical-homogeneity $[8,19,36,37]$.

The general procedure used to compute the spectrum of a quantity $\phi(x, y, t)$ within the mixing layer $x \in\left[\ell_{s}, \ell_{b}\right]$ of width $h(t)=\ell_{b}(t)-\ell_{s}(t)$ is described in Ref. [9]. The energy associated with each Fourier mode $k$ is obtained by averaging over the mixing layer to obtain the one-dimensional energy spectrum

$$
E_{\phi \phi}(k, t)=\frac{1}{h(t)} \int_{\ell_{s}(t)}^{\ell_{b}(t)} \hat{\phi}(k, x, t)^{*} \hat{\phi}(k, x, t) d x,
$$

where the superscript ${ }^{*}$ denotes complex conjugation and $\hat{\phi}(k, x, t)=\frac{1}{2 \pi} \int_{-\infty}^{\infty} \phi(x, y, t) \exp (-i k y) d y$ is the Fourier coefficient of the $2 \pi$-periodic function $\phi(x, y, t)$ in the $y$ direction. Numerically, the discrete (sine) cosine transform is used to compute the Fourier coefficients using the (anti)symmetry property of the underlying data in the spanwise direction. In the results presented below, all modes above the Nyquist wave number $\pi / \Delta x$ are neglected [38], and $k$ is in units of $\mathrm{cm}^{-1}$. The spectrum $E_{\phi \phi}(k, t)$ provides the energy content of all of the scales in the autocorrelation of $\phi$ as a function of time. The characteristic scale of a structure with wave number $k$ is $\sim 1 / k$. The time evolution of statistics

$$
E_{\phi \phi}(t)=\int_{0}^{\infty} E_{\phi \phi}(k, t) d k
$$

is also considered.

\section{B. Local and global mixing properties}

Quantities previously used in the analysis of RayleighTaylor mixing [7,33] are adapted here to the twodimensional Richtmyer-Meshkov instability, including mixing profiles and parameters, production and mixing fractions, energy spectra, and statistics [9].

\section{Mixing and mixing fraction profiles}

To simplify the comparison of the profiles across the mixing layer at different times, the streamwise coordinate is recentered by the midpoint between the bubble and spike location $x_{\text {mid }}(t)$, and divided by the mixing layer width $h(t)$. Thus the mixing layer extent is mapped to $\left[x-x_{\text {mid }}(t)\right] / h(t)$ $\in[-0.5,0.5]$, where $[-0.5,0]$ is the air (acetone) side and $[0,0.5]$ is the $\mathrm{SF}_{6}$ side, which facilitates the analysis of selfsimilarity.

a. Mole fraction profile. Consider the mole fraction

$$
X(x, y, t)=\frac{m(x, y, t) M_{1}}{m(x, y, t) M_{1}+[1-m(x, y, t)] M_{2}}
$$

averaged over the spanwise direction $y, \bar{X}(x, t)$. The fluids have constant densities $\rho_{1}$ and $\rho_{2}>\rho_{1} ; M_{1}$ and $M_{2}$ are the molecular weights of the air(acetone) and $\mathrm{SF}_{6}$, respectively, and $m$ is the mass fraction of $\mathrm{SF}_{6}$. The mole fraction profile $\bar{X}(x, t)$ gives the relative mass distribution of the two fluids within the mixing layer. If the two fluids are uniformly distributed over the entire domain, as expected in complete homogeneous mixing, then $\bar{X}=0.5$.

The mole fraction profiles shown in Fig. 10 at $1 \mathrm{~ms}$ time intervals increase from $\bar{X}=0$ in the air (acetone) to $\bar{X}=1$ in the $\mathrm{SF}_{6}$. The increasing profile width shows the spatial spreading of the mixing layer in time. The profiles show the varying mass distribution inside the layer prior to reshock. At the initial time, $\bar{X}$ increases monotonically as the initial interface is slightly diffused. After the initial shock passage, $\bar{X}$ widens and becomes nonmonotonic, with a peak developing within the air (acetone) that moves left and increases with time. This is due to spikes of $\mathrm{SF}_{6}$ penetrating into the air (acetone), forming the characteristic rollups resulting from the entrainment of additional $\mathrm{SF}_{6}$ within the air (acetone). The bubbles of air (acetone) "rise" in the $\mathrm{SF}_{6}$, causing an overall decrease in $\bar{X}$ in the $\mathrm{SF}_{6}$. Reshock compresses $\bar{X}$ between 6 and $7 \mathrm{~ms}$, and also generates additional structure reflected in the distribution of $\bar{X}$ (now with localized peaks). The layer growth slows at later times, the localized peaks decrease in magnitude, and $\bar{X}$ begins to approach 0.5 on average, indicating a well-mixed distribution of mass within the layer. 

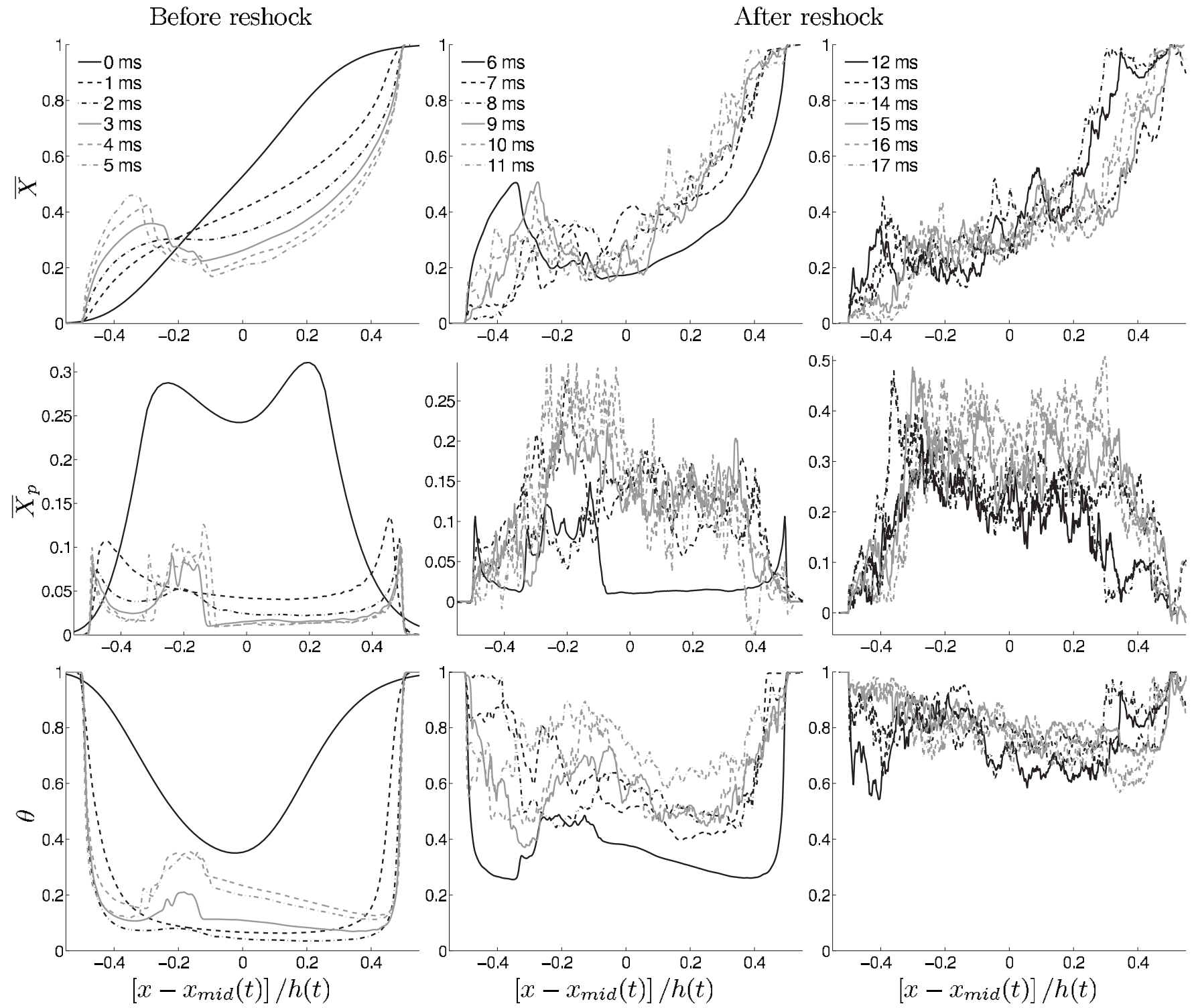

FIG. 10. The time evolution of the mole fraction profile $\bar{X}$ (top row), product mole fraction profile $\bar{X}_{p}$ (middle row), and mixing fraction profile $\theta$ (bottom row) across the mixing layer at $1 \mathrm{~ms}$ intervals. The profiles before reshock $(0-5 \mathrm{~ms})$ are shown in the first column, and the profiles following reshock $(6-17 \mathrm{~ms})$ are shown in the second and third columns.

b. Product mole fraction profile. A quantitative measure of mixing can be defined using $X(x, y, t)$ as follows. Suppose that the two fluids undergo a fast kinetic reaction, so that the amount of "product" produced is $[33,39]$

$$
X_{p}(x, y, t)= \begin{cases}\frac{X(x, y, t)}{X_{s}} & \text { for } X \leqslant X_{s} \\ \frac{1-X(x, y, t)}{1-X_{s}} & \text { for } X>X_{s}\end{cases}
$$

where $X_{s}=1 / 2$ (indicating that the product is composed of one mole of each "reactant"), and is limited by the amount of reactant (either heavy or light fluid). The product mole fraction profile $\bar{X}_{p}(x, t) \in[0,1]$ is a measure of how well mixed the reactants are. Here, numerically induced mixing is a surrogate for a chemical reaction.

The time evolution of the product mole fraction profile $\bar{X}_{p}$ is shown in Fig. 10. The initial diffusion layer is well mixed, with a rapid decrease in $\bar{X}_{p}$ between 0 and $1 \mathrm{~ms} ; \bar{X}_{p}$ broadens and rapidly develops sharp cusps at the mixing layer edges $x / h(t) \approx \pm 0.5$ as the heavy fluid spikes penetrate into the lighter fluid and the light fluid bubbles "rise" in the lighter fluid (and the fluids become less mixed). At these early times in the instability evolution, $\bar{X}_{p}$ is nearly symmetric, with values $\approx 0.04-0.06$ over most of the layer. A pronounced asymmetry develops for $t>2 \mathrm{~ms}$ : as the rollups form, additional peaks within the air (acetone) develop, corresponding to well-mixed fluid in the cores. Reshock significantly increases $\bar{X}_{p}$, as additional fine-scale structures form; 
$\bar{X}_{p}$ oscillates following reshock, which persists to late times. At late times the fluids are more mixed and larger peaks develop in the $\mathrm{SF}_{6}$, corresponding to the arrival of multiple reflected waves that increase mixing at this end of the layer. The gases are less mixed in the region closer to the air (acetone) than in the region closer to the $\mathrm{SF}_{6}$.

c. Mixing fraction profile. Averaging the volume fractions of fluids $r=1$ and 2,

$$
f_{r}(x, y, t) \equiv \frac{m_{r}(x, y, t) / \rho_{r}}{m_{1}(x, y, t) / \rho_{1}+m_{2}(x, y, t) / \rho_{2}},
$$

over the spanwise direction gives the volume fraction profiles $\bar{f}_{r}(x, t)$. The molecular mixing fraction $\theta(x, t)$ is then obtained from the volume fraction profiles and the average of their product $[7,40]$,

$$
\theta(x, t)=\frac{\overline{f_{1} f_{2}}}{\bar{f}_{1} \bar{f}_{2}},
$$

where $f_{2}=1-f_{1}$. When $\theta=0$ (i.e., $\overline{f_{1} f_{2}}=0$ ), the two fluids are completely segregated, and when $\theta=1$ (i.e., $\overline{f_{1} f_{2}}=\bar{f}_{1} \bar{f}_{2}$ ), they are completely molecularly mixed. Prior to reshock, the evolution of $\theta$ in Fig. 10 indicates increased mixing toward the air (acetone) side, corresponding to the formation of the rollup with a well-mixed core. Additional mixed structures within the rollup are indicated by the peaks at 5 and $6 \mathrm{~ms}$. At reshock $(\approx 6.4 \mathrm{~ms})$, the layer is compressed by the passage of the shock [which continues into the air (acetone)] and is "pulled back" on the $\mathrm{SF}_{6}$ side by the reflected rarefaction. This effect is captured by $\theta$, which is larger on the air (acetone) side and smaller on the $\mathrm{SF}_{6}$ side. Following reshock, the layer contains complex, disordered, well-mixed structures, as measured by a large value of $\theta$ across the layer (varying from approximately 0.6 to 1 ). On average, the largest values of $\theta$ across the entire layer are attained at the latest time, with the degree of mixedness increasing, as indicated by $\theta$ approaching unity. This "molecular mixing" is induced by stirring and by the numerical diffusion in the algorithm, rather than by molecular processes. Comparison of the profiles at $6-11 \mathrm{~ms}$ to those at $12-17 \mathrm{~ms}$ shows that $\bar{X}, \bar{X}_{p}$, and $\theta$ become approximately self-similar at late times, with intermittent variations across the layer.

\section{Production and mixing fractions}

The total chemical product $P_{t}(t)$ and maximum chemical product $P_{m}(t)$ are $[33,39]$

$$
\begin{gathered}
P_{t}(t)=\frac{1}{h(t)} \int_{\ell_{s}(t)}^{\ell_{b}(t)} \bar{X}_{p} d x, \\
P_{m}(t)=\frac{1}{h(t)} \int_{\ell_{s}(t)}^{\ell_{b}(t)} X_{p}(\bar{X}) d x,
\end{gathered}
$$

where $\ell_{b}$ and $\ell_{s}$ are the positions of the bubble and spike, respectively. In general $P_{m}(t)>P_{t}(t)$, and is the amount of
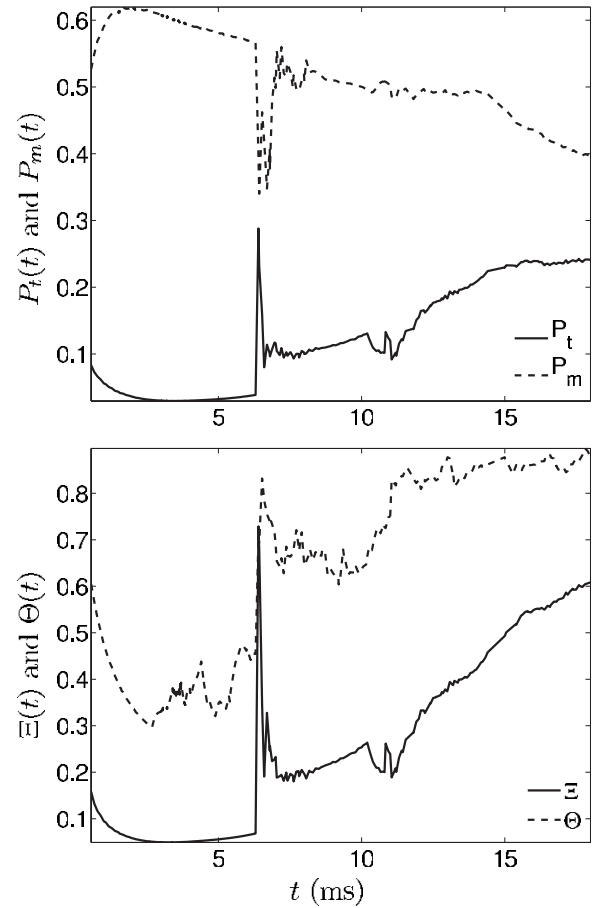

FIG. 11. The time evolution of the production fractions $P_{t}(t)$ and $P_{m}(t)$ (top) and mixing fractions $\Xi(t)$ and $\Theta(t)$ (bottom).

product obtained if both reactants were homogeneously mixed. The mixing fractions are $[7,33,40]$

$$
\Xi(t)=\frac{P_{t}(t)}{P_{m}(t)}, \quad \Theta(t)=\frac{\int_{\ell_{s}(t)}^{\ell_{b}(t)} \overline{f_{1} f_{2}} d x}{\int_{\ell_{s}(t)}^{\ell_{b}(t)} \bar{f}_{1} \bar{f}_{2} d x} .
$$

Note that $\Xi$ is a measure of the total product formed relative to the product that would be formed if all entrained fluid were completely mixed. Increasing $\Xi$ and $\Theta$ correspond to more complete mixing.

The time evolution of the global production quantities $P_{t}$ and $P_{m}$ is shown in Fig. 11. Before reshock, $P_{t}$ increases, indicating an increase in mixing, while $P_{m}$ decreases. The decrease in $P_{m}$ indicates that the maximum molar product decreases due to the bubble (which creates large, unmixed structures within the mixing layer). During reshock, the mixing layer is compressed, inducing additional mixing as measured by $P_{t}$. Following reshock, $P_{t}$ increases rapidly, indicating significantly increased mixing. As the mixing progresses, large unmixed structures form, decreasing $P_{m}$.

The time evolution of the mixing fractions $\Xi$ and $\Theta$ shown in Fig. 11 provides qualitatively similar information, with $\Theta(t)>\Xi(t)$ for all time. As the fluids mix following the initial shock passage, the mixing fractions decrease and then increase. Immediately after reshock, the layer is compressed, causing a well-mixed region to form, as shown by the sharply peaked values of $\Xi$ and $\Theta$. This well-mixed region is characterized by the rapid production of small-scale flow structures by reshock, as shown in Sec. III. Following reshock, $\Xi$ and $\Theta$ increase faster than before reshock, indicat- 

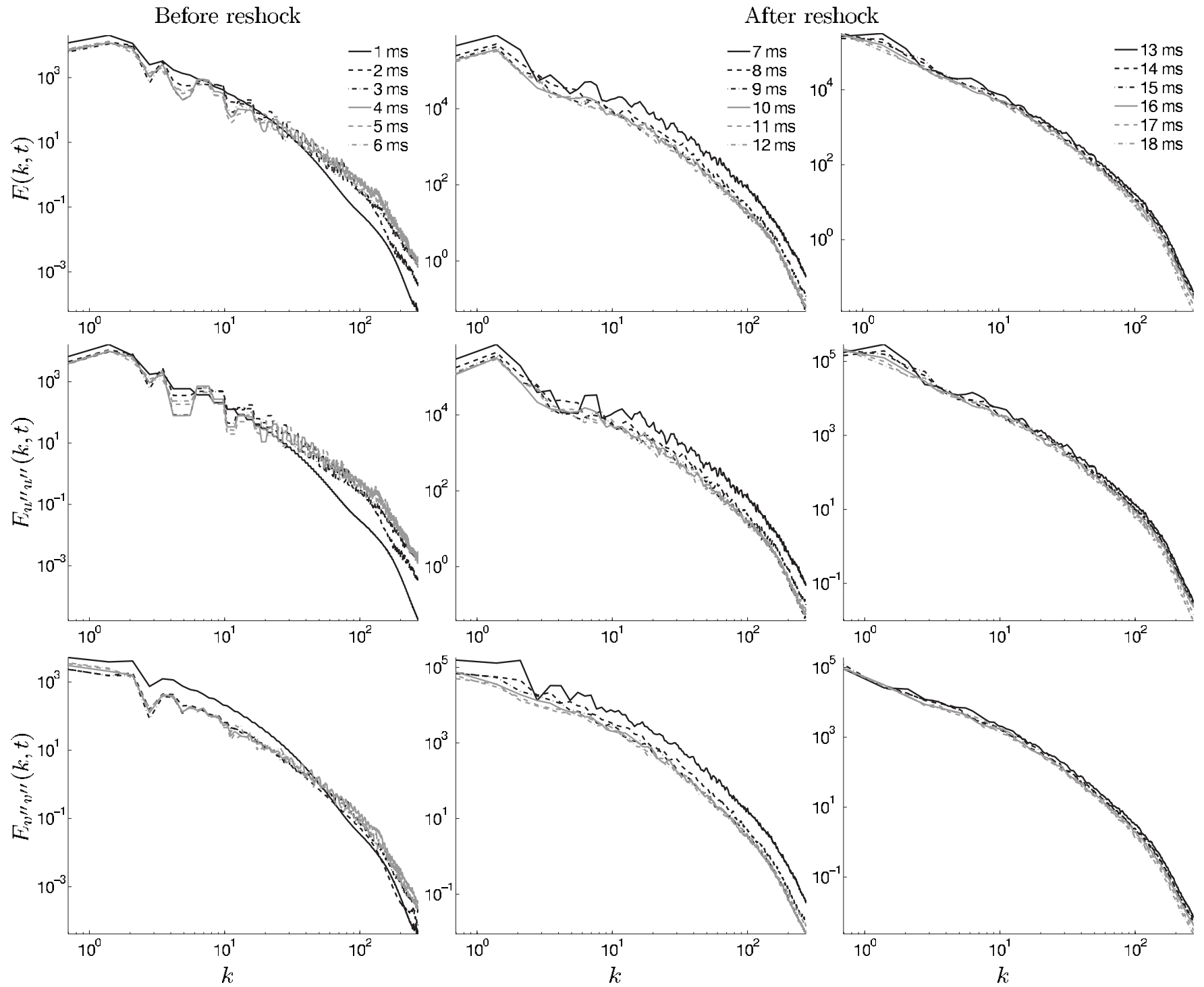

FIG. 12. The time evolution of the total, streamwise, and spanwise fluctuating kinetic energy spectra $E(k, t)$ (top row), $E_{u^{\prime \prime} u^{\prime \prime}}(k, t)$ (middle row), and $E_{v^{\prime \prime} v^{\prime \prime}}(k, t)$ (bottom row), respectively, at $1 \mathrm{~ms}$ intervals (in units of $\mathrm{cm}^{3} / \mathrm{s}^{2}$ ). The spectra before reshock are shown in the first column and the spectra after reshock are shown in the second and third columns.

ing that reshock increases the mixing rate, as expected physically. Subsequently, the mixing layer undergoes a rapid growth as the bubbles and spikes invert, causing the overall mixing rate to decrease rapidly over a time interval of $\approx 2 \mathrm{~ms}$. Following this decrease, the formation of complex flow features with rollups again increases the mixing. Note that the arrival of the reflected rarefaction wave at $\approx 11 \mathrm{~ms}$ and compression wave at $\approx 15 \mathrm{~ms}$ is reflected in the production and mixing fractions.

\section{Time evolution of energy spectra and statistics}

Fluctuating energy spectra provide a measure of the growth and decay of the energy at different scales in the flow at a given time. The energy spectra are compared here to the scalings suggested by two-dimensional turbulence phenomenology. The time evolution of statistics (integrated energy spectra) is also considered.

\section{Energy spectra}

The time evolution of the total, streamwise, and spanwise fluctuating kinetic energy spectra per unit mass $E(k, t)$ $=E_{u^{\prime \prime} u^{\prime \prime}}(k, t)+E_{v^{\prime \prime} v^{\prime \prime}}(k, t), E_{u^{\prime \prime} u^{\prime \prime}}(k, t)$, and $E_{v^{\prime \prime} v^{\prime \prime}}(k, t)$, respectively, is shown in Fig. 12. The oscillations at small and intermediate $k$ at early times and following reshock are damped out at late times, as indicated by the more smoothly decaying spectra at large $t$. Reshock sharply increases the spectra between 6 and $7 \mathrm{~ms}$ by exciting a wide range of scales, thereby imparting additional energy into the mixing layer. For $t>8 \mathrm{~ms}$, the spectra decay very slowly in time. The streamwise fluctuating kinetic energy spectrum $E_{u^{\prime \prime} u^{\prime \prime}}(k, t)$ is peaked at $k \approx 1.5 \mathrm{~cm}^{-1}$, while the peak of the spanwise fluctuating kinetic energy spectrum $E_{v^{\prime \prime} v^{\prime \prime}}(k, t)$ corresponds to the largest scale of the flow.

The time evolution of the ratio of the streamwise and spanwise fluctuating kinetic energy spectra 

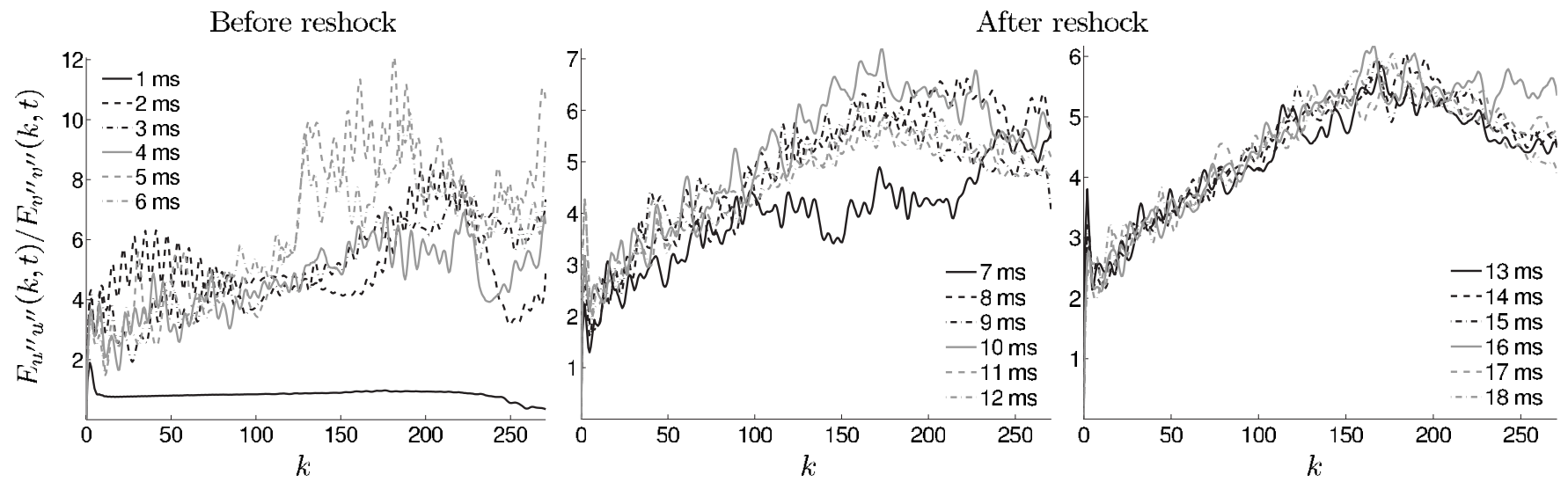

FIG. 13. The time evolution of the ratio of the streamwise and spanwise fluctuating kinetic energy spectra $E_{u^{\prime \prime} u^{\prime \prime}}(k, t) / E_{v^{\prime \prime} v^{\prime \prime}}(k, t)$ before and after reshock at $1 \mathrm{~ms}$ intervals. The ratios before reshock are shown in the first column and the ratios after reshock are shown in the second and third columns.

$E_{u^{\prime \prime} u^{\prime \prime}}(k, t) / E_{v^{\prime \prime} v^{\prime \prime}}(k, t)$ is shown in Fig. 13. As expected, there is more energy content in the streamwise than spanwise velocity fluctuations, i.e., the energy spectra are anisotropic. The evolution of $E(k, t)$ is dominated by that of $E_{u^{\prime \prime} u^{\prime \prime}}(k, t)$. The ratio is nearly a constant for all scales at $1 \mathrm{~ms}$. At later times prior to reshock, the ratio increases at intermediate and large $k$, with similar increases at reshock. At late times $(t$ $\geqslant 13 \mathrm{~ms}$ ) the ratio changes very little with time, with a value $\approx 2$ at the smallest $k$ increasing nearly linearly to $\approx 6$ at $k$ $\approx 170 \mathrm{~cm}^{-1}$, and then decreasing to $\approx 4.5$ at $k \approx 270 \mathrm{~cm}^{-1}$. Reshock, the arrival of the reflected rarefaction wave at $\approx 11 \mathrm{~ms}$, and the compression wave at $\approx 15 \mathrm{~ms}$ decrease the ratio, indicating that these waves excite more spanwise fluctuations relative to streamwise fluctuations. At late times, the ratio indicates that spectral isotropy (i.e., a ratio of unity) is not achieved, as the streamwise fluctuating kinetic energy spectrum contains more small-scale energy than the spanwise spectrum by a factor $\approx 2-6$.

The fluctuating enstrophy density per unit mass is $\Omega(x, y, t)^{\prime \prime}=\left[\omega(x, y, t)^{\prime \prime}\right]^{2} / 2 ;$ the time evolution of the fluctuating enstrophy spectrum $E_{\omega^{\prime \prime} \omega^{\prime \prime}}(k, t)$ is shown in Fig. 14. In a homogeneous flow, the fluctuating enstrophy and kinetic energy spectra are related by $E_{\omega^{\prime \prime} \omega^{\prime \prime}}(k, t)=k^{2} E(k, t)$, so that the peak of $E_{\omega^{\prime \prime} \omega^{\prime \prime}}(k, t)$ is weighted toward smaller scales than that of $E(k, t)$. The fluctuating enstrophy spectrum is less steep than the fluctuating kinetic energy spectrum. As in the case of $E(k, t)$, reshock primarily amplifies $E_{\omega^{\prime \prime} \omega^{\prime \prime}}(k, t)$ but does not change its qualitative shape. The spectra decay very slowly at late times.

The time evolution of the density and pressure variance spectra, $E_{\rho^{\prime} \rho^{\prime}}(k, t)$ and $E_{p^{\prime} p^{\prime}}(k, t)$, is also shown in Fig. 14. The density variance spectrum varies very little with time, indicating that density fluctuations are not as strongly affected by reshock as other quantities. Moreover, the magnitude of $E_{\rho^{\prime} \rho^{\prime}}(k, t)$ is very small for all times, indicating that the density fluctuations are very small and the flow is nearly incompressible: additional numerical evidence supports this conclusion in Richtmyer-Meshkov unstable flows [15,16]. The spectrum also shows the interaction of reflected waves with the mixing layer, which have a profound effect on
$E_{p^{\prime} p^{\prime}}(k, t)$. The pressure variance spectrum increases very rapidly upon reshock at $\approx 6.4 \mathrm{~ms}$, and also exhibits a rapid increase at $\approx 11 \mathrm{~ms}$ when the reflected rarefaction interacts with the mixing layer. Both $E_{\rho^{\prime} \rho^{\prime}}(k, t)$ and $E_{p^{\prime} p^{\prime}}(k, t)$ decay very slowly at late times.

To determine the scales at which the baroclinic vorticity production is large, consider the baroclinic vorticity production variance spectrum $E_{\mathcal{P P}}(k, t)$ shown in Fig. 14. This spectrum is large and nearly constant over all scales for $t$ $>2 \mathrm{~ms}$ and decreases rapidly at the smallest scales. The spectrum increases rapidly after $2 \mathrm{~ms}$ (prior to reshock), consistent with additional baroclinic production at the interface where the rollup occurs. Prior to reshock, $E_{\mathcal{P P}}(k, t)$ is highly oscillatory. A large increase occurs at reshock, followed by a rapid decay. A further increase occurs between 11 and $12 \mathrm{~ms}$, corresponding to the arrival of the reflected rarefaction. At late times, $E_{\mathcal{P P}}(k, t)$ decays at a significantly faster rate than the other spectra.

\section{Comparison to classical two-dimensional turbulence inertial subrange scalings}

The fluctuating kinetic energy, enstrophy, and density and pressure variance spectra are compared here to the inertial subrange scalings predicted by two-dimensional turbulence phenomenology [41-46]. It is of interest to explore whether these scalings apply in the reshocked two-dimensional Richtmyer-Meshkov unstable flow considered here, in which the numerical Reynolds number attains values $O\left(10^{4}\right)-O\left(10^{5}\right)$ based on two different definitions (see Ref. [9]). This phenomenology applies to incompressible and isotropic turbulence; here it is assumed that after reshock, the flow can be regarded as nearly incompressible and approximately isotropic at late times.

For sufficiently large Reynolds numbers, the inertial subrange fluctuating kinetic energy spectrum scales as

$$
E(k, t)=K o^{\prime} \eta(t)^{2 / 3} k^{-3}, \quad k_{f} \ll k \ll k_{\eta}(t),
$$

which was originally proposed for turbulence forced at a wave number $k_{f}$, with kinetic energy and enstrophy injection rates $\epsilon$ and $\eta$, respectively. Here, $K o^{\prime}$ is the two-dimensional 

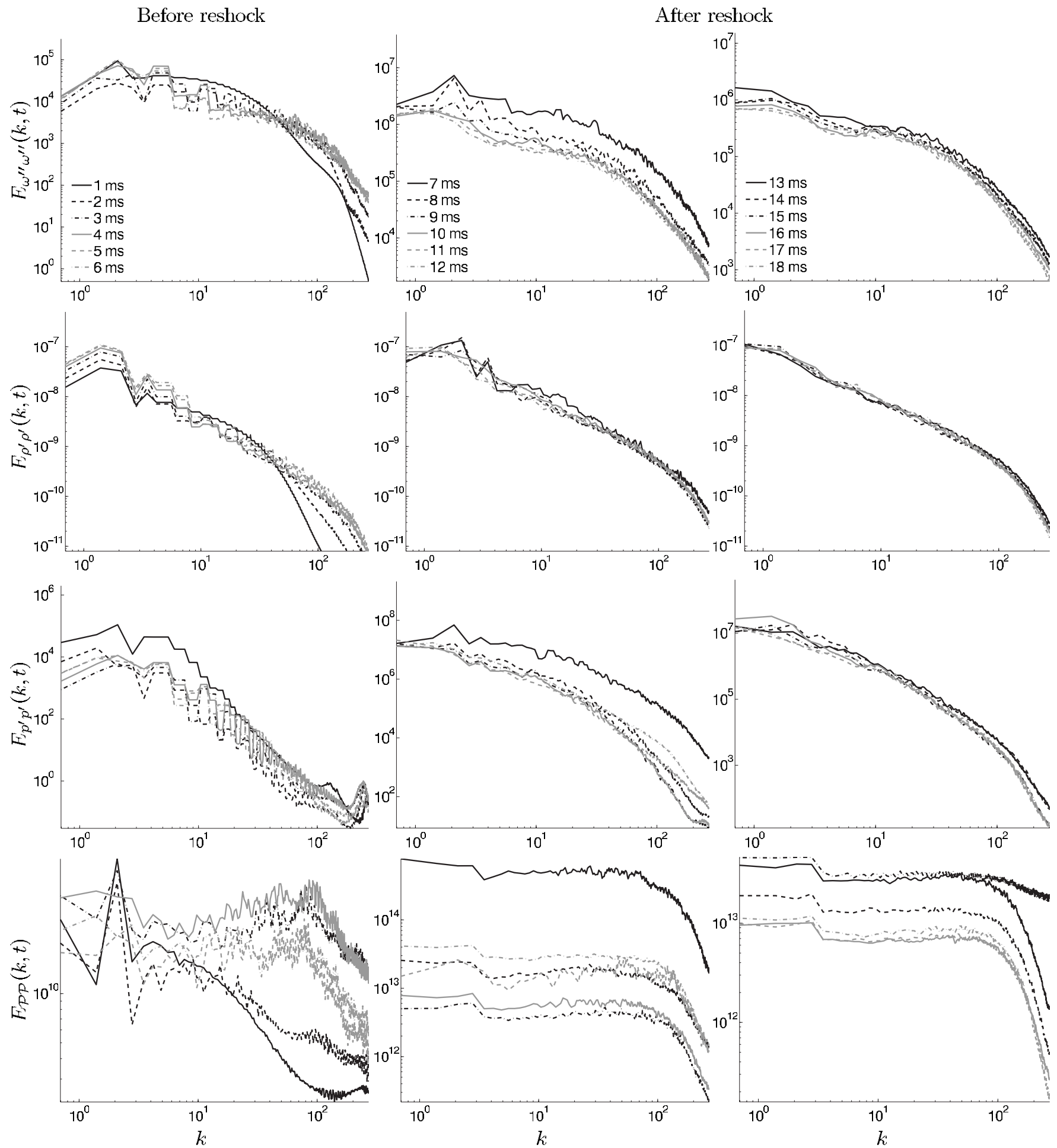

FIG. 14. The time evolution of the fluctuating enstrophy spectrum $E_{\omega^{\prime \prime} \omega^{\prime \prime}}(k, t)$ (in units of $\left.\mathrm{cm} / \mathrm{s}^{2}\right)$, density variance spectrum $E_{\rho^{\prime} \rho^{\prime}}(k, t)$ (in units of $\mathrm{g}^{2} / \mathrm{cm}^{5}$ ), pressure variance spectrum $E_{p^{\prime} p^{\prime}}(k, t)$ (in units of $\operatorname{bar}^{2} \mathrm{~cm}$ ), and baroclinic vorticity production variance spectrum $E_{\mathcal{P} p}(k, t)$ (in units of $\mathrm{cm} / \mathrm{s}^{4}$ ) at $1 \mathrm{~ms}$ intervals. The spectra before reshock are shown in the first column and the spectra after reshock are shown in the second and third columns.

Kolmogorov constant and $k_{\eta}(t)=\left[\eta(t) / \nu^{3}\right]^{1 / 6}$ is the enstrophy dissipation wave number (with $\nu$ the kinematic viscosity). A subsequent logarithmic modification to the $k^{-3}$ law explicitly depending on $k_{f}$ was required for self-consistency [47]. The inertial subrange enstrophy spectrum corresponding to Eq. (26) is

$$
E_{\omega^{\prime} \omega^{\prime}}(k, t)=K o^{\prime} \eta(t)^{2 / 3} k^{-1}
$$



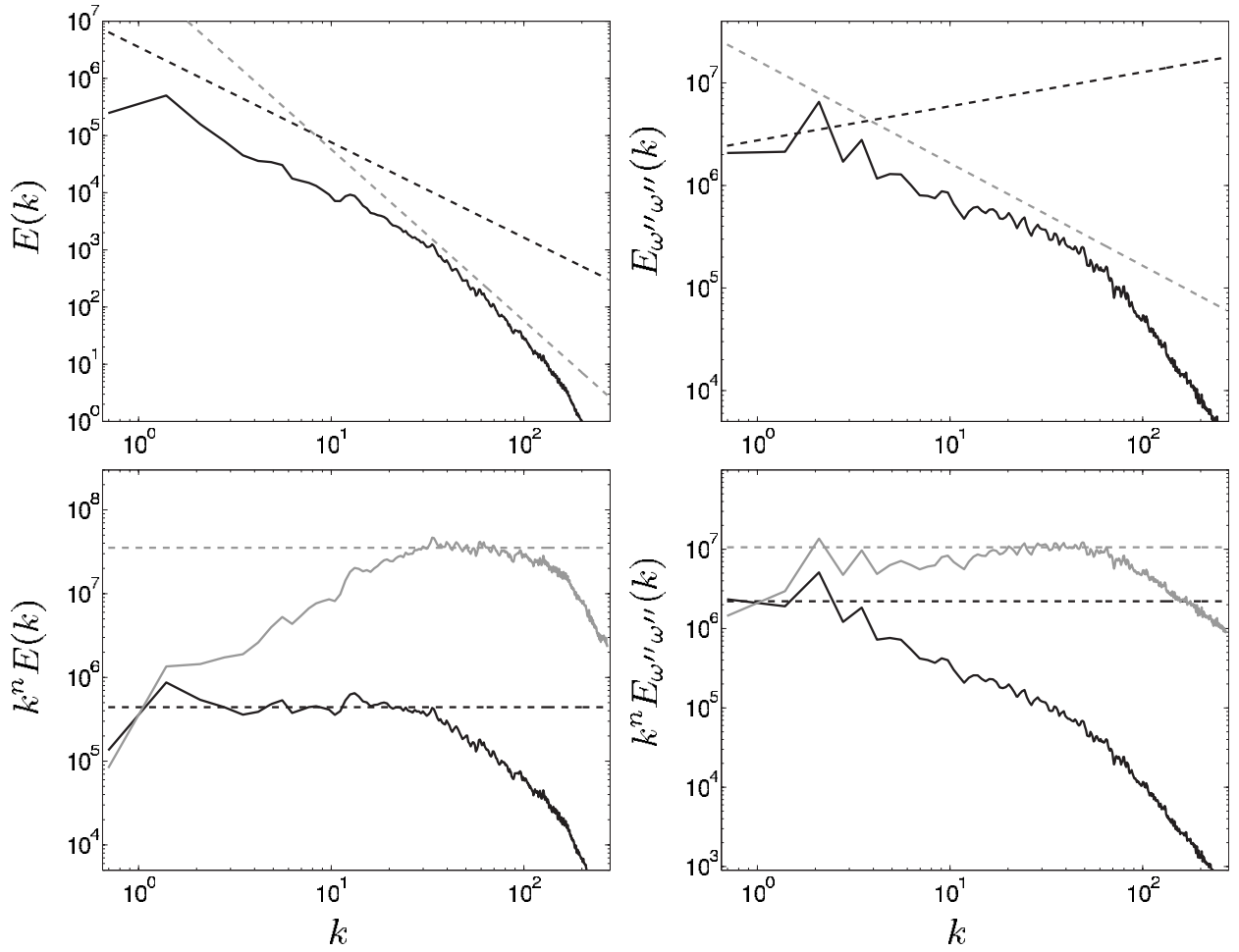

FIG. 15. The fluctuating kinetic energy spectrum $E(k)$ and compensated spectrum $k^{n} E(k)$ (left column) at $8 \mathrm{~ms}$ showing a $k^{-5 / 3}$ decay (black dotted line) for small $k$ with $K o^{\prime \prime} \epsilon^{2 / 3}=4.4127$ $\times 10^{5} \mathrm{~cm}^{4 / 3} / \mathrm{s}^{2}$ and an approximate $k^{-3}$ decay (gray dotted line) for large $k$ with $K o^{\prime} \eta^{2 / 3}=3.5301$ $\times 10^{7} \mathrm{~s}^{-2}$. The fluctuating enstrophy spectrum $E_{\omega^{\prime \prime} \omega^{\prime \prime}}(k)$ and compensated spectrum $k^{n} E_{\omega^{\prime \prime} \omega^{\prime \prime}}(k)$ (right column) at $8 \mathrm{~ms}$ showing an approximate $k^{1 / 3}$ growth (black dotted line) for small $k$ with $K o^{\prime \prime} \epsilon^{2 / 3}=2.2063 \times 10^{6} \mathrm{~cm}^{4 / 3} / \mathrm{s}^{2}$ and an approximate $k^{-1}$ decay (gray dotted line) for large $k$ with $K o^{\prime} \eta^{2 / 3}=1.059 \times 10^{7} \mathrm{~s}^{-2}$.
These are the spectra in the enstrophy cascade subrange in which the turbulent kinetic energy and enstrophy flux are $\Pi(k, t) \approx 0$ and $\Pi_{\Omega}(k, t) \approx \eta(t)$, respectively.

In the inverse kinetic energy cascade subrange in which $\Pi(k, t) \approx \epsilon(t)$ and $\Pi_{\Omega}(k, t) \approx 0$, the inertial subrange kinetic energy spectrum for small wave numbers is

$$
E(k, t)=K o^{\prime \prime} \epsilon(t)^{2 / 3} k^{-5 / 3}, \quad k \ll k_{f}
$$

with a different Kolmogorov constant $K o^{\prime \prime}$. The corresponding inertial subrange enstrophy spectrum is

$$
E_{\omega^{\prime} \omega^{\prime}}(k, t)=K o^{\prime \prime} \epsilon(t)^{2 / 3} k^{1 / 3}, \quad k \ll k_{f}
$$

The above scaling laws are a consequence of the conservation of kinetic energy and enstrophy in two-dimensional, nearly inviscid, very high Reynolds number turbulent flows.

In a nearly incompressible flow, density fluctuations are expected to be weakly coupled to the velocity field, so that the density fluctuations may be passively advected by the velocity field. If the Reynolds number is sufficiently large to support an inertial subrange, and the Schmidt number is small enough so that molecular diffusivity is negligible over a large wave number range, then there is an inertialconvective subrange in which the density variance spectrum scales as

$$
E_{\rho^{\prime} \rho^{\prime}}(k, t)=\mathrm{Ba} \frac{\epsilon_{\rho^{\prime} \rho^{\prime}}(t)}{\epsilon(t)^{1 / 3}} k^{-5 / 3},
$$

where $\mathrm{Ba}$ is the Batchelor (or Corrsin-Obukhov) constant and $\epsilon_{\rho^{\prime} \rho^{\prime}}(t)$ is the density variance dissipation rate [48-52]. Dimensionally, the inertial subrange pressure variance spectrum in two-dimensional large Reynolds number turbulence is proportional to $k E(k, t)^{2}$, so that [53]

$$
\begin{gathered}
E_{p^{\prime} p^{\prime}}(k, t)=K o_{p}^{\prime} \bar{\rho}^{2} \eta(t)^{4 / 3} k^{-5}, \quad k_{f} \ll k \ll k_{\eta}(t), \\
E_{p^{\prime} p^{\prime}}(k, t)=K o_{p}^{\prime \prime} \bar{\rho}^{2} \epsilon(t)^{4 / 3} k^{-7 / 3}, \quad k \ll k_{f},
\end{gathered}
$$

in the enstrophy cascade and inverse kinetic energy cascade subranges, respectively, where $K o_{p}^{\prime}$ and $K o_{p}^{\prime \prime}$ are pressure Kolmogorov constants.

The present simulations only consider one statistical realization; if a set of different flow realizations were ensemble averaged, the resulting flow would appear more statistically homogeneous in the spanwise direction (and the spectra would be smoother). Here, spectra and compensated spectra are considered at two times following reshock: at $8 \mathrm{~ms}$ (shortly after reshock when the spectra have begun to relax to a quasidecaying state following the noisy transient just after reshock at $7 \mathrm{~ms}$ ), and at $18 \mathrm{~ms}$ (at late time when the effects of the shock on the flow have diminished, and the flow has relaxed to a more isotropic state). The interaction of the shock with the evolving layer during the reshock process imparts additional kinetic energy, enstrophy, and other fluctuations into the layer. Following reshock, the flow enters a slowly decaying phase during which it is possible to investigate spectral scalings instantaneously.

The fluctuating kinetic energy spectrum $E(k)$ at $8 \mathrm{~ms}$ is shown in Fig. 15, together with the expected power laws: $k^{-5 / 3}$ for the small, energy-containing wave numbers and $k^{-3}$ for larger wave numbers. Also shown are the compensated spectra used to verify the approximate scalings and the wave number extent of these scalings (less than one decade). Peng, Zabusky, and Zhang [19] also found that the densityweighted kinetic energy spectrum was consistent with a $k^{-3}$ power law in their two-dimensional piecewise-parabolic 

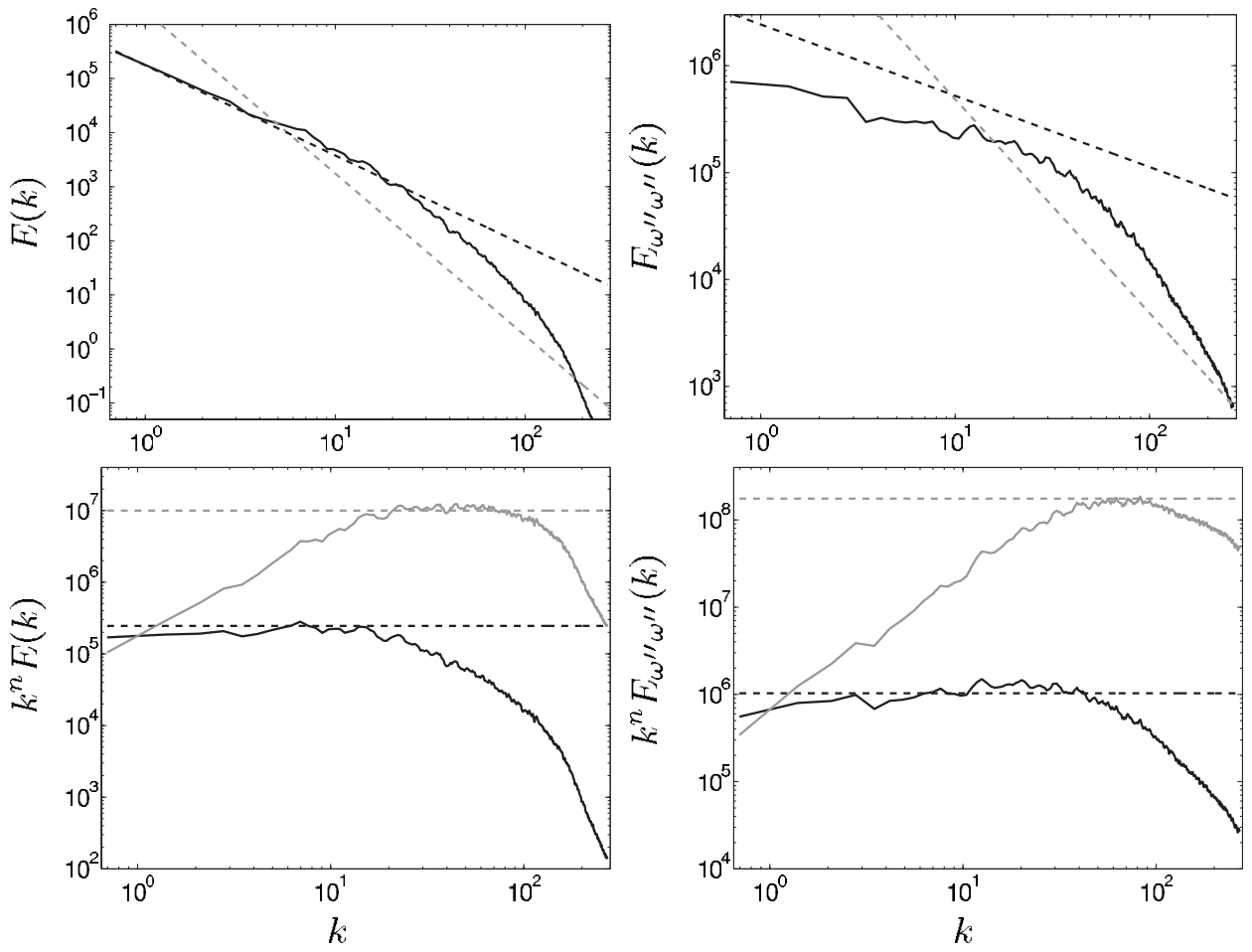

FIG. 16. The fluctuating kinetic energy spectrum $E(k)$ and compensated spectrum $k^{n} E(k)$ (left column) at $18 \mathrm{~ms}$ showing a $k^{-5 / 3}$ decay (black dotted line) for small $k$ with $K o^{\prime \prime} \epsilon^{2 / 3}=2.4546$ $\times 10^{5} \mathrm{~cm}^{4 / 3} / \mathrm{s}^{2}$ and an approximate $k^{-3}$ decay (gray dotted line) for large $k$ with $K o^{\prime} \eta^{2 / 3}=9.9937$ $\times 10^{6} \mathrm{~s}^{-2}$. The fluctuating enstrophy spectrum $E_{\omega^{\prime \prime} \omega^{\prime \prime}}(k)$ and compensated spectrum $k^{n} E_{\omega^{\prime \prime} \omega^{\prime \prime}}(k)$ (right column) at $18 \mathrm{~ms}$ showing an approximate $k^{-2 / 3}$ decay (black dotted line) for small $k$ and an approximate $k^{-2}$ decay (gray dotted line) for large $k$. method simulation of a model of the Jacobs and Krivets [54] Mach 1.3 single-mode, air/SF 6 Richtmyer-Meshkov instability experiment.

The fluctuating enstrophy spectrum $E_{\omega^{\prime \prime} \omega^{\prime \prime}}(k)$ at $8 \mathrm{~ms}$ is also shown in Fig. 15. As the vorticity is the spatial derivative of the velocity field, the fluctuating enstrophy and fluctuating kinetic energy spectra are related by $E_{\omega^{\prime \prime} \omega^{\prime \prime}}(k, t)$ $=k^{2} E(k, t)$. Consequently, the expected inertial subrange scalings for the intermediate and large wave numbers are $k^{1 / 3}$ and $k^{-1}$, respectively. The compensated spectra show a possible $k^{-1}$ scaling over less than one decade; however, there is an insufficient range of wave numbers in the largest scales to ascertain whether a $k^{1 / 3}$ scaling exists.

The fluctuating kinetic energy spectrum $E(k)$ and fluctuating enstrophy spectrum $E_{\omega^{\prime \prime} \omega^{\prime \prime}}(k)$ are shown at $18 \mathrm{~ms}$ in Fig. 16. The fluctuating kinetic energy spectrum exhibits a scaling similar to the one immediately following reshock, i.e., $k^{-5 / 3}$ for intermediate scales and $k^{-3}$ for small scales, as verified by the compensated energy spectrum. However, the fluctuating enstrophy spectrum exhibits a different scaling than expected in classical two-dimensional turbulence: a decay close to $k^{-2 / 3}$ and $k^{-2}$ is observed for intermediate and small scales, respectively, as shown by the compensated spectra. The fluctuating enstrophy scalings $k^{1 / 3}$ and $k^{-1}$ can only be expected in the presence of forcing that sustains the turbulence. In the absence of such forcing, the spectra may satisfy different scalings. In the case of the RichtmyerMeshkov instability, a transient forcing in the momentum equation, $F \sim \rho \Delta v_{1} \delta\left(t-t_{r}\right)$, is induced by the interaction of the reflected shock with the evolving mixing layer (reshock), where $\Delta v_{1}$ is the velocity jump and $t_{r}$ is the time at which reshock occurs. Following the passage of the shock, no additional forcing is provided, and the turbulence enters a quasidecay phase, in which only interactions with smaller amplitude waves occur: this results in different scaling laws for the late-time enstrophy spectrum than expected classically.

The density and pressure variance spectra, $E_{\rho^{\prime} \rho^{\prime}}(k)$ and $E_{p^{\prime} p^{\prime}}(k)$, are shown following reshock at $8 \mathrm{~ms}$ and at late time $(18 \mathrm{~ms})$ in Figs. 17 and 18 , respectively. The density variance spectrum shows an approximate $k^{-5 / 3}$ scaling for intermediate-to-large $k$, which is further verified by the compensated spectrum. The pressure variance spectrum $E_{p^{\prime} p^{\prime}}(k)$ shows a scaling of $k^{-7 / 3}$ over less than a decade of wave numbers at late time, which is also verified by the compensated spectrum. A $k^{-5}$ decay of $E_{p^{\prime} p^{\prime}}(k)$ at large $k$ is inconclusive. The $k^{-5 / 3}$ scaling for the density variance spectrum and the $k^{-7 / 3}$ scaling for the pressure variance spectrum may be approximately valid in the two-dimensional case considered here.

\section{Statistics}

The statistics within the mixing layer were obtained by integrating the spectra over all wave numbers. The time evolution of the streamwise, spanwise, and total fluctuating kinetic energy is shown in Fig. 19. Initially, $E_{v^{\prime \prime} v^{\prime \prime}}(t)$ is much smaller than $E_{u^{\prime \prime} u^{\prime \prime}}(t)$ as the initial shock primarily excites modes in the streamwise direction. The kinetic energy decreases following the initial shock passage, while reshock deposits additional vorticity on the interface and amplifies the kinetic energy. Reshock and the subsequent reflected wave interactions also excite spanwise velocity fluctuations, as can also be seen by considering the time evolution of the ratio $E_{u^{\prime \prime} u^{\prime \prime}}(t) / E_{v^{\prime \prime} v^{\prime \prime}}(t)$, also shown in Fig. 19. On average, this ratio is $\gtrsim 3$, with a peak value $\approx 20$ at reshock. At $18 \mathrm{~ms}$ this ratio decreases to 1.28 , showing that the contributions from the spanwise and streamwise fluctuations become com- 

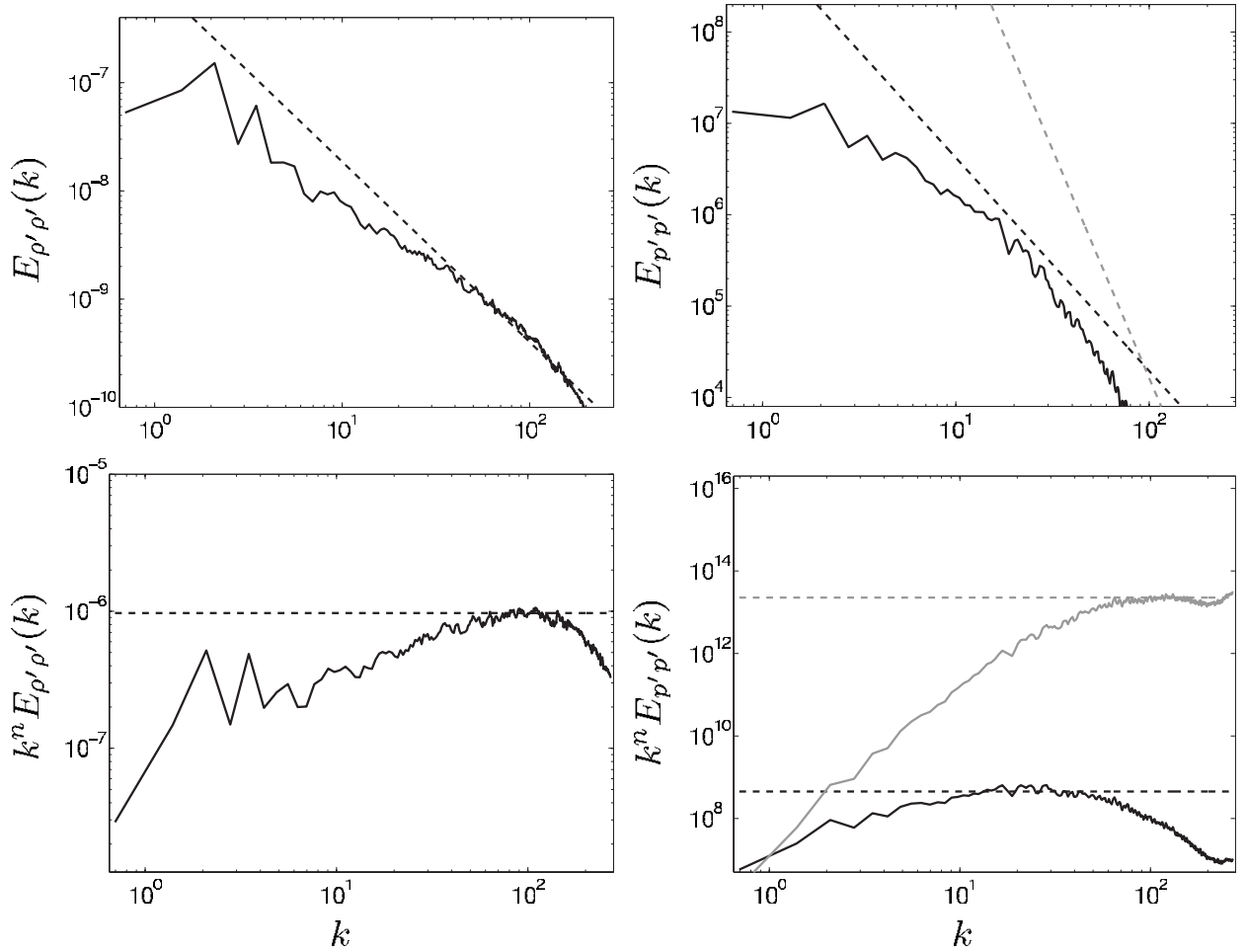

FIG. 17. The density variance spectrum $E_{\rho^{\prime} \rho^{\prime}}(k)$ and compensated spectrum $k^{n} E_{\rho^{\prime} \rho^{\prime}}(k)$ (left column) at $8 \mathrm{~ms}$ showing a very short $k^{-5 / 3}$ decay with $B a \epsilon_{\rho^{\prime} \rho^{\prime}}$ $\epsilon^{1 / 3}=9.6997 \times 10^{-7} \mathrm{~g}^{2} / \mathrm{cm}^{20 / 3}$. The pressure variance spectrum $E_{p^{\prime} p^{\prime}}(k)$ and compensated spectrum $k^{n} E_{p^{\prime} p^{\prime}}(k)$ (right column) at $8 \mathrm{~ms}$ showing a very short $k^{-7 / 3}$ decay (black dotted line) for small $k$ with $K o_{p}^{\prime \prime} \rho^{2} \epsilon^{4 / 3}=4.5218 \times 10^{8}$ $\mathrm{g}^{2} /\left(\mathrm{cm}^{10 / 3} \mathrm{~s}^{4}\right)$ and a very short $k^{-5}$ decay (gray dotted line) for large $k$ with $K o_{p}^{\prime} \bar{\rho}^{2} \eta^{4 / 3}=2.2609 \times 10^{13}$ $\mathrm{g}^{2} /\left(\mathrm{cm}^{6} \mathrm{~s}^{4}\right)$. parable at late times, and indicating an approach of the flow to statistical (but not spectral) isotropy at late times.

The fluctuating enstrophy $E_{\omega^{\prime \prime} \omega^{\prime \prime}}(t)$ in Fig. 20 increases significantly during reshock. Following reshock, $E_{\omega^{\prime \prime} \omega^{\prime \prime}}(t) \mathrm{de}-$ cays, but the arrival of the reflected waves compensates for the decrease, and a nearly constant value is observed for $8-11 \mathrm{~ms}$. Finally, after the interaction of the reflected rarefaction with the mixing layer at $\approx 11 \mathrm{~ms}, E_{\omega^{\prime \prime} \omega^{\prime \prime}}(t)$ begins to decay once again.
The density variance $E_{\rho^{\prime} \rho^{\prime}}(t)$ in Fig. 20 is not as significantly affected by reshock or by other reflected waves as the other statistics, and remains nearly constant in time. The pressure variance $E_{p^{\prime} p^{\prime}}(t)$ is much more sensitive, exhibiting peaks as waves interact with the mixing layer. The first peak at $\approx 6.4 \mathrm{~ms}$ corresponds to the arrival of the reflected shock, the second peak at $\approx 11 \mathrm{~ms}$ corresponds to the arrival of the reflected rarefaction, and the third peak at $\approx 15 \mathrm{~ms}$ corresponds to the arrival of the reflected compression wave. The
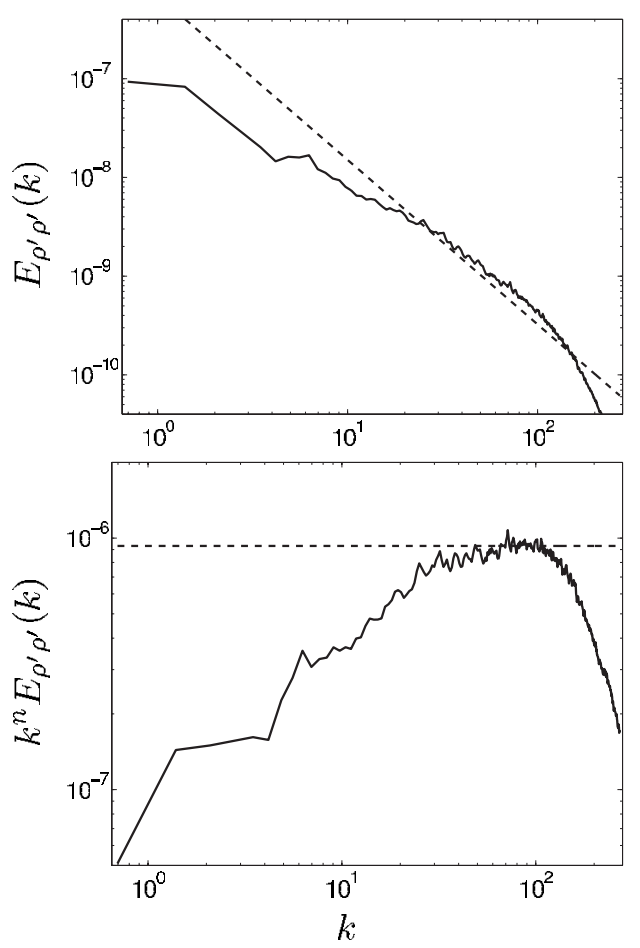
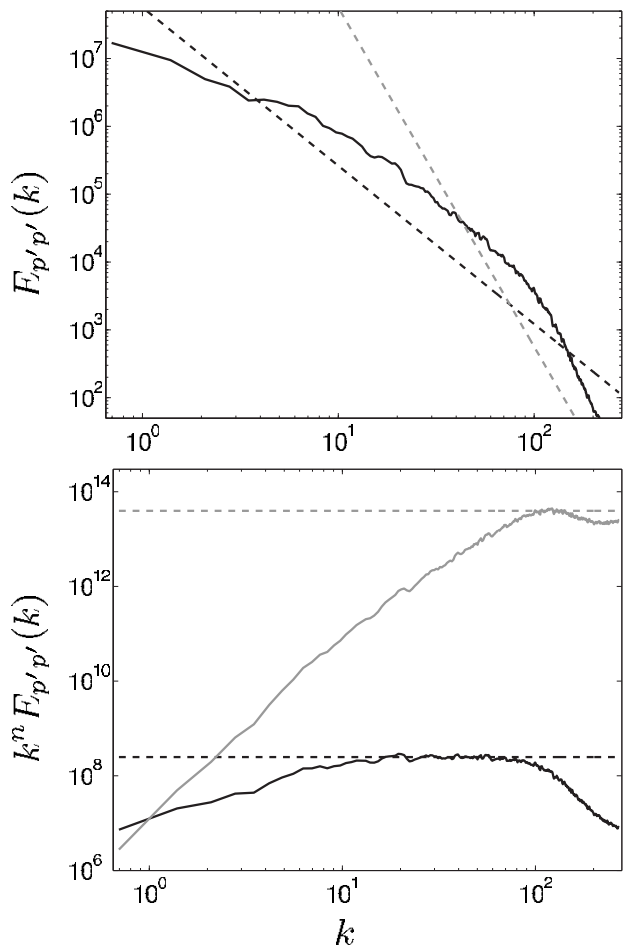

FIG. 18. The density variance spectrum $E_{\rho^{\prime} \rho^{\prime}}(k)$ and compensated spectrum $k^{n} E_{\rho^{\prime} \rho^{\prime}}(k) \quad$ (left column) at $18 \mathrm{~ms}$ showing a very short $k^{-5 / 3}$ decay with $B a \epsilon_{\rho^{\prime} \rho^{\prime}} /$ $\epsilon^{1 / 3}=9.3085 \times 10^{-7} \mathrm{~g}^{2} / \mathrm{cm}^{20 / 3}$. The pressure variance spectrum $E_{p^{\prime} p^{\prime}}(k)$ and compensated spectrum $k^{n} E_{p^{\prime} p^{\prime}}(k)$ (right column) at $18 \mathrm{~ms}$ showing an approximate $k^{-7 / 3}$ decay (black dotted line) for small $k$ with $K o_{p}^{\prime \prime} \bar{\rho}^{2} \epsilon^{4 / 3}=2.4885$ $\times 10^{8} \mathrm{~g}^{2} /\left(\mathrm{cm}^{10 / 3} \mathrm{~s}^{4}\right)$ and a very short $k^{-5}$ decay (gray dotted line) for large $k$ with $K o_{p}^{\prime} \bar{\rho}^{2} \eta^{4 / 3}$ $=3.9598 \times 10^{13} \mathrm{~g}^{2} /\left(\mathrm{cm}^{6} \mathrm{~s}^{4}\right)$. 

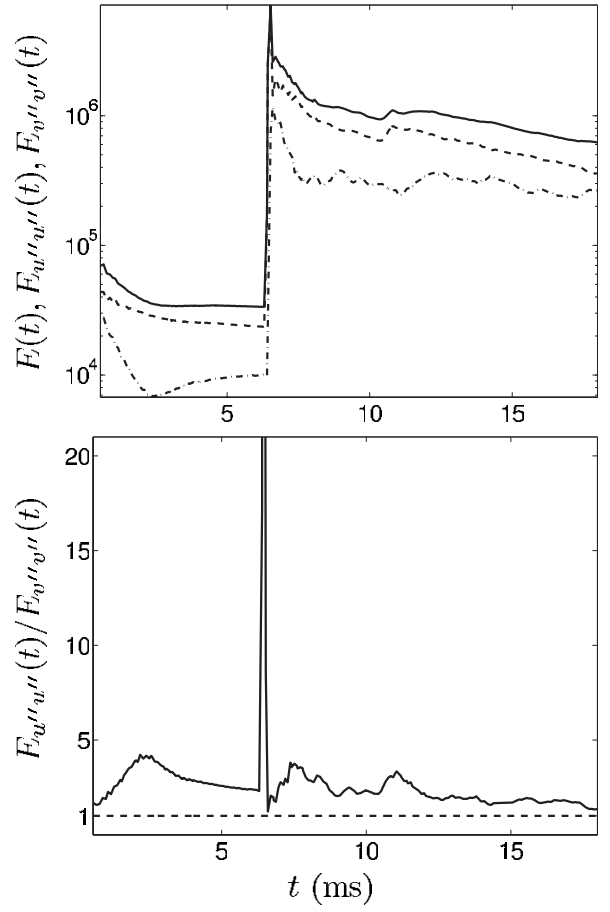

FIG. 19. The time evolution of the fluctuating kinetic energy $E(t)$ (solid line) and its streamwise and spanwise components $E_{u^{\prime \prime} u^{\prime \prime}}(t)$ and $E_{v^{\prime \prime} v^{\prime \prime}}(t)$ (dashed and dot-dashed lines, respectively) (in units of $\mathrm{cm}^{2} / \mathrm{s}^{2}$ ) (top), and the time evolution of the ratio of the streamwise and spanwise component of the fluctuating kinetic energy $E_{u^{\prime \prime} u^{\prime \prime}}(t) / E_{v^{\prime \prime} v^{\prime \prime}}(t)$ (bottom).

pressure variance is the only statistic considered that captures the effect of this third interaction. With the exception of $E_{\rho^{\prime} \rho^{\prime}}(t)$, all of the other statistics decrease sharply following reshock. With the reflecting boundary condition at the end wall, the simulation does not achieve a purely decaying state at late times: later in Sec. VI E, the reflecting boundary condition is changed to outflow at the end wall in order to remove the effects of reflected waves following reshock.

The baroclinic vorticity production variance $E_{\mathcal{P P}}(t)$ is also shown in Fig. 20. Prior to reshock, $E_{\mathcal{P P}}(t)$ increases between 3.5 and $4 \mathrm{~ms}$, as the vortex rolls up. As the rollup develops, $E_{\mathcal{P P}}(t)$ is large as a result of the density gradient between the two fluids, and later decreases as the fluids mix. Reshock sharply increases the production, followed by a rapid decay after the shock passes through the interface. At $\approx 11 \mathrm{~ms}$, $E_{\mathcal{P P}}(t)$ increases corresponding to the arrival of the reflected rarefaction, followed by a decay; $E_{\mathcal{P P}}(t)$ does not increase upon the arrival of the reflected compression wave.

\section{Comparison of mixing quantities and spectra immediately after and before reshock}

As in the case of the interaction of a shock with a turbulent flow [55-57], reshock of the evolving RichtmyerMeshkov mixing layer amplifies quantities characterizing turbulence and mixing. This amplification is investigated here by comparing quantities at $t=t_{r}^{-}=6.38$ and $t=t_{r}^{+}$ $=6.58 \mathrm{~ms}$, immediately prior to and following reshock, respectively. The mole fraction profile $\bar{X}$, product mole fraction profile $\bar{X}_{p}$, and the mixing fraction profile $\theta$ prior to and following reshock are shown in Fig. 21 as a function of $[x$ $\left.-x_{\text {mid }}(t)\right] / h(t)$. The mole fraction profile shows that reshock compresses the interface, shifting mass from the $\mathrm{SF}_{6}$ side to the air (acetone) side. The product mole fraction profile shows that reshock accelerates mixing at the diffuse interfaces, including in the cores of the vortex rollups: this results in increased overall mixing, as shown by the increase in $\theta$.
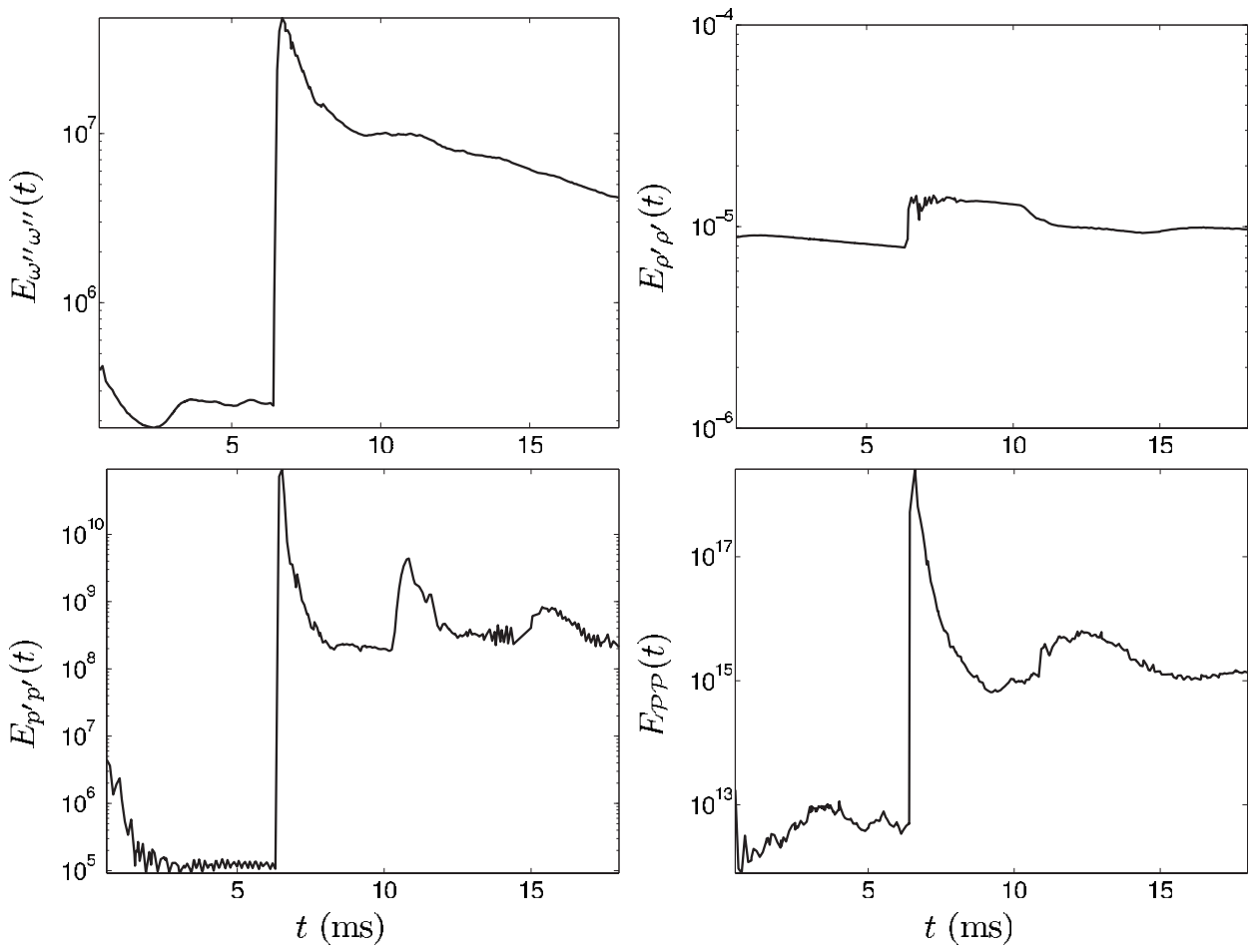

FIG. 20. The time evolution of the fluctuating enstrophy $E_{\omega^{\prime \prime} \omega^{\prime \prime}}(t)$ (in units of $\mathrm{s}^{-2}$ ), density variance $E_{\rho^{\prime} \rho^{\prime}}(t)$ (in units of $\mathrm{g}^{2} / \mathrm{cm}^{6}$ ), pressure variance $E_{p^{\prime} p^{\prime}}(t)$ (in units of $\mathrm{bar}^{2}$ ), and baroclinic vorticity production variance $E_{\mathcal{P P}}(t)$ (in units of $\mathrm{s}^{-4}$ ). 

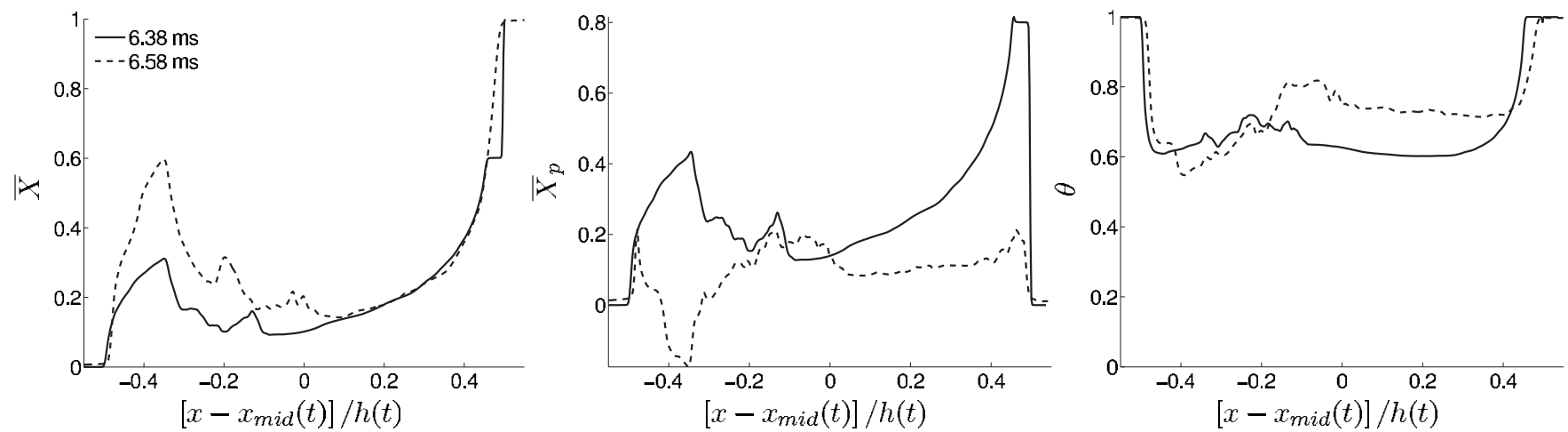

FIG. 21. Comparison of the mole fraction and production fraction profiles, $\bar{X}$ and $\bar{X}_{p}$, and of the mixing fraction $\theta$ before reshock at $t_{r}^{-}=6.38 \mathrm{~ms}$ (solid line) and after reshock at $t_{r}^{+}=6.58 \mathrm{~ms}$ (dashed line).

The fluctuating kinetic energy spectrum $E(k, t)$ and its streamwise and spanwise components, $E_{u^{\prime \prime} u^{\prime \prime}}(k, t)$ and $E_{v^{\prime \prime} v^{\prime \prime}}(k, t)$, are shown in Fig. 22. The ratio of the spectra after and before reshock quantifies the amplification in energy as a function of $k$. The spectra are amplified most at the intermediate and small scales. Reshock sharply increases the energy content: the increase in the total and streamwise spectra is nearly uniform for small $k$, with an increase for intermediateto-large $k$, as indicated by the ratio after and before reshock (also shown in Fig. 22). The spanwise component $E_{v^{\prime \prime} v^{\prime \prime}}(k, t)$ increases nearly uniformly from small to large $k$, as indicated by the increasing ratio. Reshock primarily excites spanwise fluctuations, as the ratio is considerably larger for $E_{v^{\prime \prime} v^{\prime \prime}}(k, t)$ than for $E_{u^{\prime \prime} u^{\prime \prime}}(k, t)$. The ratio of the streamwise and spanwise fluctuating kinetic energy spectra, $E_{u^{\prime \prime} u^{\prime \prime}}(k, t) / E_{v^{\prime \prime} v^{\prime \prime}}(k, t)$, is shown in Fig. 23 after and before reshock: this ratio decreases with $k$ following reshock. The ratio is of $O(1)$ at intermediate $k\left(k>5 \mathrm{~cm}^{-1}\right)$ and approaches $\approx 0.3$ for large $k$ : reshock significantly increases the spanwise fluctuations to a level comparable to the streamwise fluctuations at the largest scales. This "equilibration" is only transient, as the spanwise component dominates shortly after reshock once inversion occurs.

The fluctuating enstrophy, pressure variance, and baroclinic vorticity production variance spectra in Figs. 24 and 25 also sharply increase following reshock. The largest amplification of $E_{\omega^{\prime \prime} \omega^{\prime \prime}}(k, t)$ occurs at intermediate to small $k$. The density variance spectrum $E_{\rho^{\prime} \rho^{\prime}}(k, t)$ is not significantly amplified compared to the other spectra, and shows an average amplification of $\approx 3$. By contrast, the pressure variance
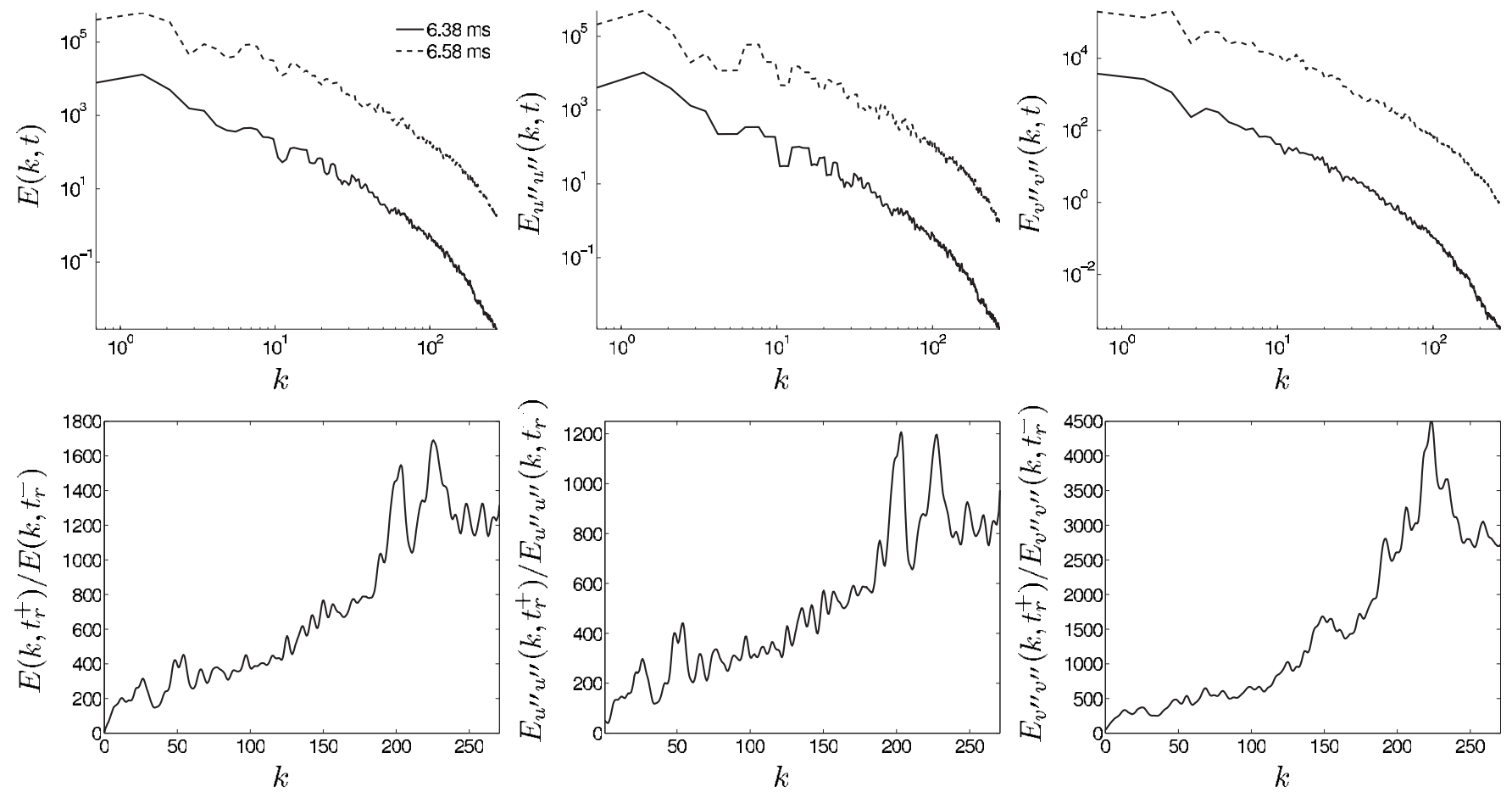

FIG. 22. Comparison of the fluctuating kinetic energy spectrum $E(k, t)$ and its streamwise and spanwise components, $E_{u^{\prime \prime} u^{\prime \prime}}(k, t)$ and $E_{v^{\prime \prime} v^{\prime \prime}}(k, t)$, respectively (in units of $\mathrm{cm}^{3} / \mathrm{s}^{2}$ ), before reshock at $t_{r}^{-}=6.38 \mathrm{~ms}$ and after reshock at $t_{r}^{+}=6.58 \mathrm{~ms}$ (top row). The ratios of these spectra after and before reshock are also shown (bottom row). 

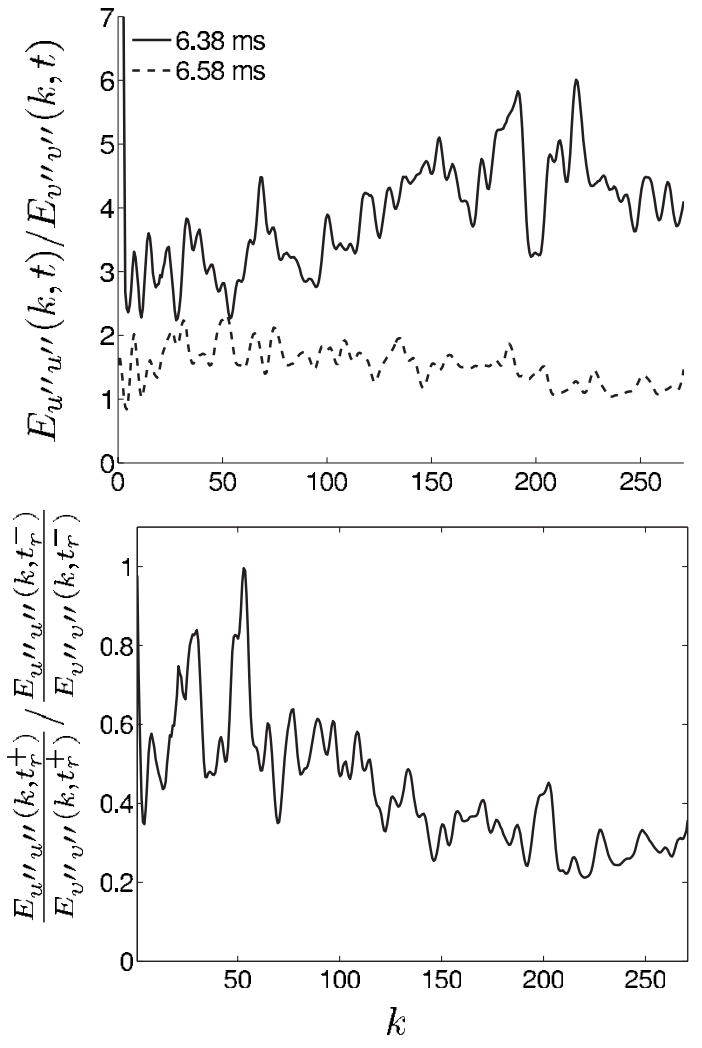

FIG. 23. Comparison of the ratio of the streamwise and spanwise fluctuating kinetic energy spectrum $E_{u^{\prime \prime} u^{\prime \prime}}(k, t) / E_{v^{\prime \prime} v^{\prime \prime}}(k, t)$ before reshock at $t_{r}^{-}=6.38 \mathrm{~ms}$ and after reshock at $t_{r}^{+}=6.58 \mathrm{~ms}$ (top). The ratio $\left[E_{u^{\prime \prime} u^{\prime \prime}}\left(k, t_{r}^{+}\right) / E_{v^{\prime \prime} v^{\prime \prime}}\left(k, t_{r}^{+}\right)\right] /\left[E_{u^{\prime \prime} u^{\prime \prime}}\left(k, t_{r}^{-}\right) / E_{v^{\prime \prime} v^{\prime \prime}}\left(k, t_{r}^{-}\right)\right]$after and before reshock is also shown (bottom). spectrum undergoes the most amplification, having a peak of $\approx 1.4 \times 10^{7}$ for $k \approx 110 \mathrm{~cm}^{-1}$. The baroclinic vorticity production variance spectrum also shows an amplification of $\approx 8 \times 10^{5}$ for small $k$, with the amplification decreasing at larger $k$.

\section{E. The effects of reflected waves on the mixing dynamics}

Following reshock, the rarefaction formed during reshock at $\approx 11 \mathrm{~ms}$ interacts with the mixing layer, causing the formation of a compression wave, which subsequently interacts with the layer at $\approx 15 \mathrm{~ms}$. Here, the boundary condition at the right end of the computational domain (corresponding to the end wall of the test section) is changed from reflecting to outflow immediately following reshock to allow the reflected rarefaction to exit the computational domain, so that no further interactions of waves with the layer occur. The purpose of this is to investigate the properties of mixing in the decay regime, as distinguished from those in the quasidecay regime occurring when waves interact with the evolving interface following reshock. Another objective is to understand the effects of a reflected rarefaction on the evolution of a mixing layer in the same spirit that the study of reshock investigates the effects of a shock wave on an evolving mixing layer. The results are from simulations with resolution of 256 points per initial perturbation wavelength (the WENO9M simulation in Ref. [9]).

A comparison of the mixing layer width, production fractions, and mixing fractions is shown in Fig. 26 for reflecting and outflow boundary conditions. As expected, the quantities overlap from the time of reshock $\approx 6.4 \mathrm{~ms}$ until $\approx 11 \mathrm{~ms}$
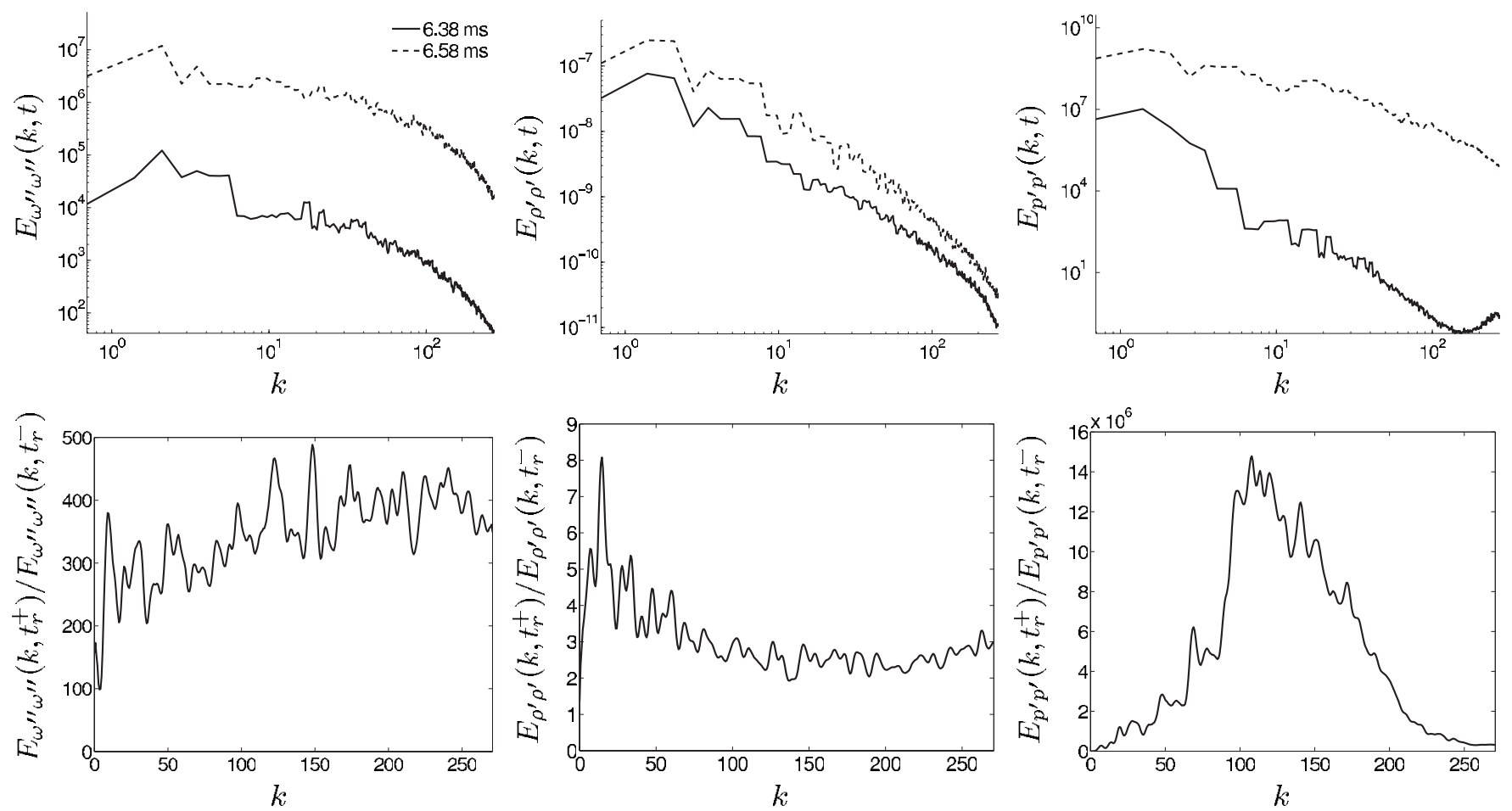

FIG. 24. Comparison of the fluctuating enstrophy spectrum $E_{\omega^{\prime \prime} \omega^{\prime \prime}}(k, t)$ (in units of $\mathrm{cm} / \mathrm{s}^{2}$ ), density variance spectrum $E_{\rho^{\prime} \rho^{\prime}}(k, t)$ (in units of $\mathrm{g}^{2} / \mathrm{cm}^{5}$ ), and pressure variance spectrum $E_{p^{\prime} p^{\prime}}(k, t)$ (in units of $\operatorname{bar}^{2} \mathrm{~cm}$ ) before reshock at $t_{r}^{-}=6.38 \mathrm{~ms}$ and after reshock at $t_{r}^{+}$ $=6.58 \mathrm{~ms}$ (top row). The ratios of the spectra after and before reshock are also shown (bottom row). 

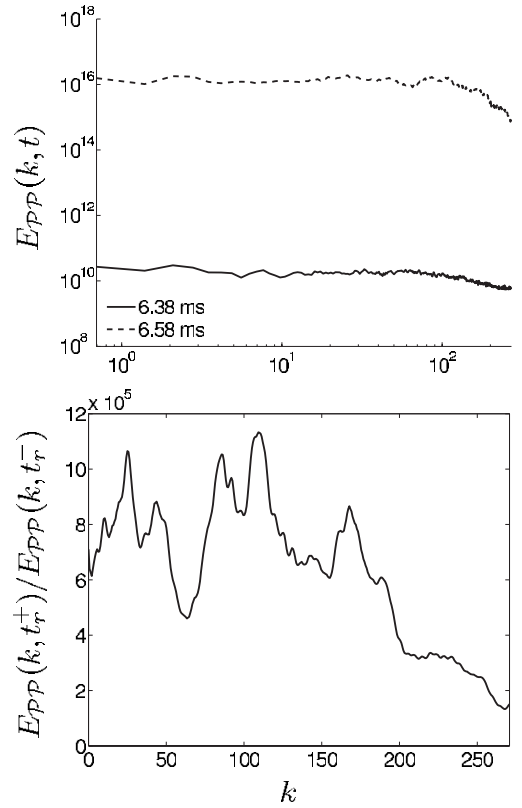

FIG. 25. Comparison of the baroclinic vorticity production variance spectrum $E_{\mathcal{P P}}(k, t)$ (in units of $\mathrm{cm} / \mathrm{s}^{4}$ ) before reshock at $t_{r}^{-}$ $=6.38 \mathrm{~ms}$ and after reshock at $t_{r}^{+}=6.58 \mathrm{~ms}$ (top). The ratio of the spectra after and before reshock is also shown (bottom).

when the reflected rarefaction interacts with the mixing layer. Generally, quantities computed with the outflow boundary condition are smoother compared to those computed with the reflecting boundary condition, as a consequence of the absence of wave interactions with the layer.

A comparison of the spectra obtained with the outflow and reflecting boundary conditions is shown in Figs. 27-30 at $12 \mathrm{~ms}$, following the arrival of the reflected rarefaction wave and at $18 \mathrm{~ms}$. When the ratios are shown, the quantity computed in the reflecting boundary condition case is divided by the corresponding quantity in the outflow case. The reflected rarefaction has a modest effect on the fluctuating kinetic energy (and its streamwise and spanwise components), fluctuating enstrophy, and pressure and density variance spectra. The reflected rarefaction contributes to approximately a factor of 2 difference in the energy content, which is significantly less than the corresponding contribution by energy deposition during reshock. However, the effects on the ratio of the spanwise and streamwise components in Fig. 28 are significant, indicating that the reflected rarefaction excites spanwise velocity fluctuations and contributes to the late-time isotropization of the velocity field. The reflected rarefaction increases $E_{\mathcal{P P}}(k, t)$ in Fig. 30 by an order of magnitude.

A comparison of the evolution of the statistics obtained with reflecting and outflow boundary conditions is shown in Figs. 31 and 32 . The fluctuating kinetic energy per unit mass $E(t)$ and its components $E_{u^{\prime \prime} u^{\prime \prime}}(t)$ and $E_{v^{\prime \prime} v^{\prime \prime}}(t)$ are shown in Fig. 31. In the outflow case, the energy is not increased by the reflected waves, and therefore decays. The reflected rarefaction increases the energy in the spanwise component, contributing to the statistical isotropization of the flow. In the outflow case, both components retain their separation, as shown by the ratio of the streamwise and spanwise components in Fig. 31. Statistical isotropy is approached faster at late time in the reflecting than in the outflow case.

The evolution of the fluctuating enstrophy $E_{\omega^{\prime \prime} \omega^{\prime \prime}}(t)$ in Fig. 32 is very similar for the reflecting and outflow cases, indicating that the reflected waves do not increase $E_{\omega^{\prime \prime} \omega^{\prime \prime}}(t)$ significantly. However, $E_{\rho^{\prime} \rho^{\prime}}(t)$ corresponding to the outflow case is greater than $E_{\rho^{\prime} \rho^{\prime}}(t)$ corresponding to the reflecting case, indicating that the reflected rarefaction decreases the density fluctuations. The pressure variance $E_{p^{\prime} p^{\prime}}(t)$ indicates that the pressure fluctuations are nearly constant in the absence of reflected waves. By contrast, the fluctuations in the reflecting case undergo a transient increase following the interaction with reflected waves, and approach the nearly constant value corresponding to the outflow case shortly thereafter. The baroclinic vorticity production variance $E_{\mathcal{P P}}(t)$ decays in the absence of the reflected rarefaction.

\section{DISCUSSION AND CONCLUSIONS}

Presented here is a comprehensive investigation of the mixing properties before and after reshock and at late times, obtained from two-dimensional ninth-order WENO simulations using initial and boundary conditions and flow geometry modeling the test section in the Mach 1.21 Collins and Jacobs air (acetone) $/ \mathrm{SF}_{6}$ shock tube experiment. The details of the simulations are discussed in Ref. [2], which considered the growth of the perturbation amplitude into the non-
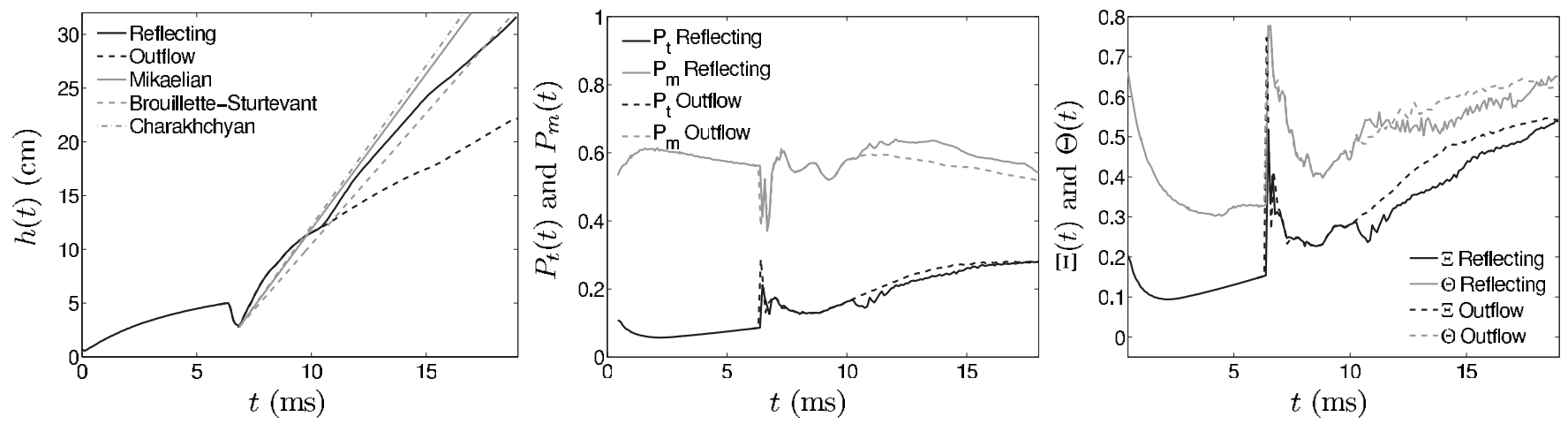

FIG. 26. Comparison of the time evolution of the mixing layer width $h(t)$, production fractions $P_{t}(t)$ and $P_{m}(t)$, and mixing fractions $\Xi(t)$ and $\Theta(t)$ for reflecting and outflow boundary conditions. 

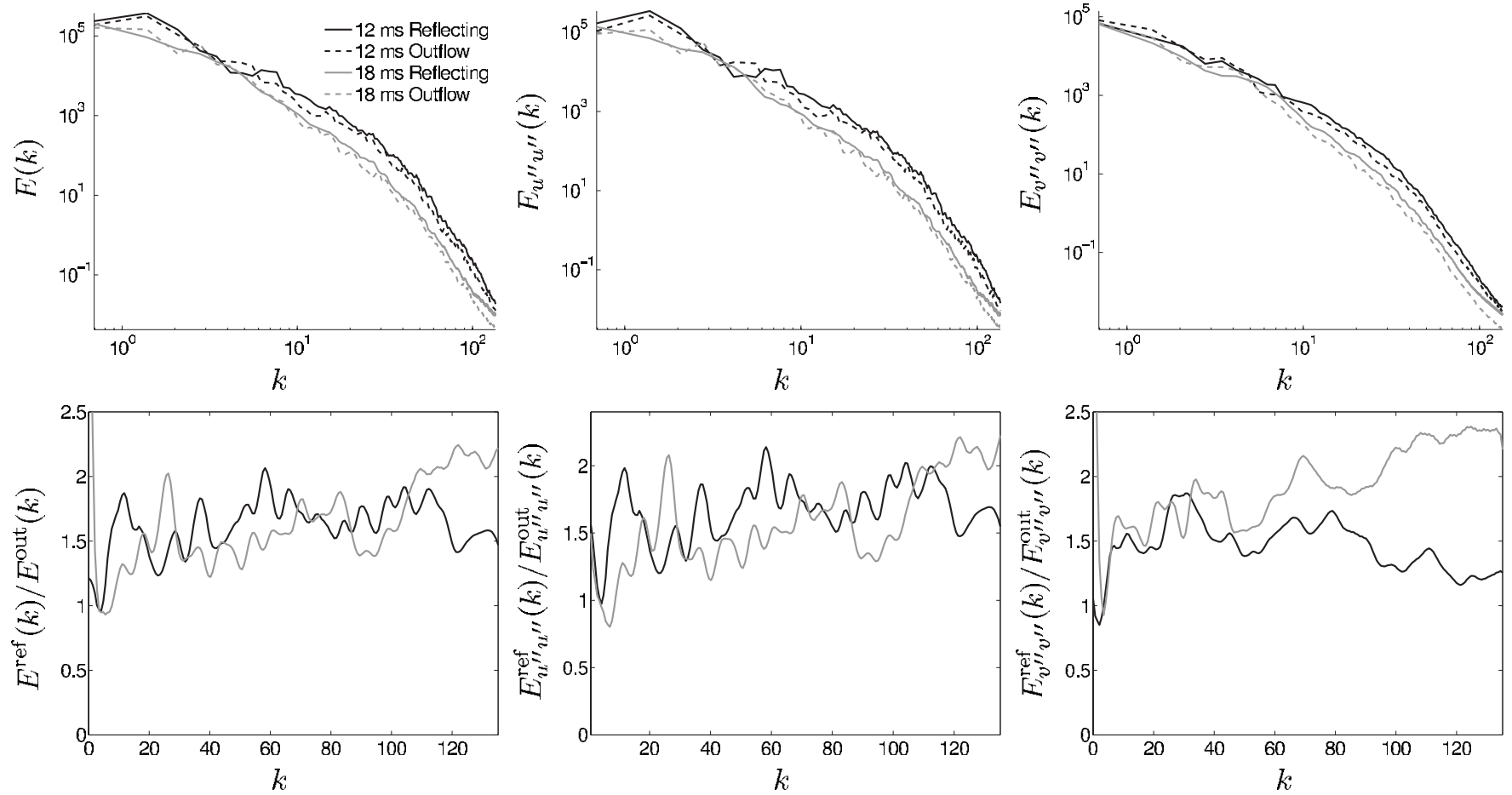

FIG. 27. Comparison of the fluctuating kinetic energy spectrum $E(k)$ and its streamwise and spanwise components, $E_{u^{\prime \prime} u^{\prime \prime}}(k)$ and $E_{v^{\prime \prime} v^{\prime \prime}}(k)$, respectively (in units of $\mathrm{cm}^{3} / \mathrm{s}^{2}$ ), at $12 \mathrm{~ms}$ (black) and $18 \mathrm{~ms}$ (gray) for reflecting and outflow boundary conditions (top row). The ratios of the spectra for the reflecting and outflow cases are also shown (bottom row).

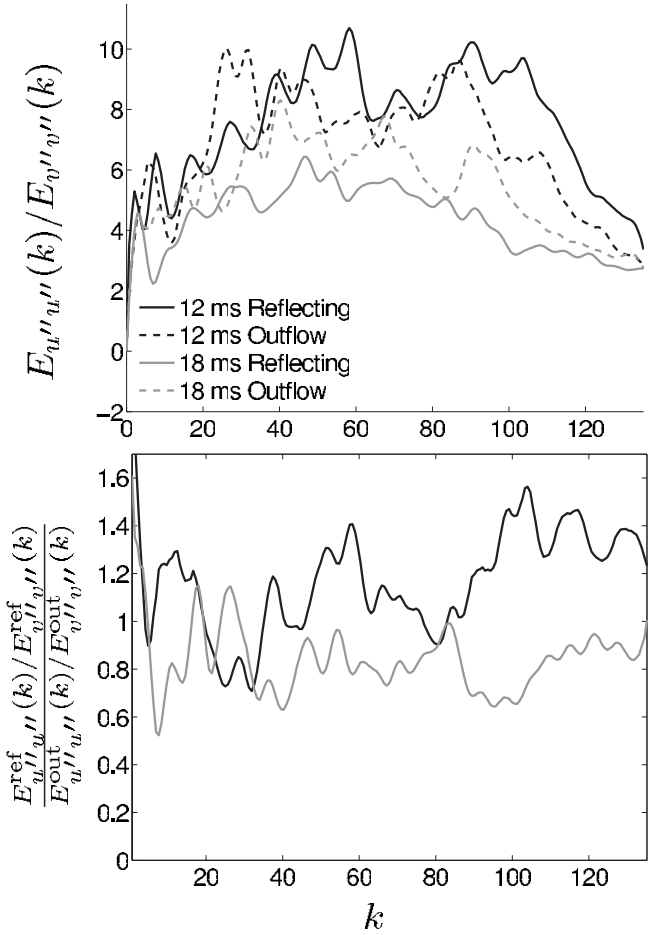

FIG. 28. Comparison of the ratio of the streamwise and spanwise fluctuating kinetic energy spectrum $E_{u^{\prime \prime} u^{\prime \prime}}(k) / E_{v^{\prime \prime} v^{\prime \prime}}(k)$ at $12 \mathrm{~ms}$ (black) and $18 \mathrm{~ms}$ (gray) for reflecting and outflow boundary conditions (top). The ratio of the spectra from the reflecting and the outflow boundary condition cases is also shown (bottom). linear regime prior to reshock. The dependence of the quantities considered here on the order of WENO flux reconstruction and grid resolution was discussed in Ref. [9].

\section{A. Phenomenology of reshock}

Density fields were used to visualize reshock, including the inversion process and the formation of complex disordered structures. The vorticity and baroclinic vorticity production fields were also used to investigate vorticity production during reshock, and the deposition of vorticity of opposite sign on the interface, which drives the inversion. Simulated density Schlieren fields were used to visualize the complex wave interactions occurring during reshock, i.e., the reflected and transmitted waves, and the focusing effects. The following qualitative picture emerges for the rollup dynamics: the misalignment of the density and pressure gradient during the rollup causes vorticity deposition of opposite sign on the interface. This vorticity is responsible for the formation of large-scale structures as the rollup develops, resulting in the formation of complex structures.

\section{B. Baroclinic interfacial circulation deposition}

To quantify the baroclinic mechanism driving the evolution of the Richtmyer-Meshkov instability, the circulation deposited on the interface by the initial shock was shown to agree well with the Samtaney-Zabusky model and with linear instability theory. The absolute values of the positive and negative circulation components increase following the shock interaction, consistent with the physical picture of 

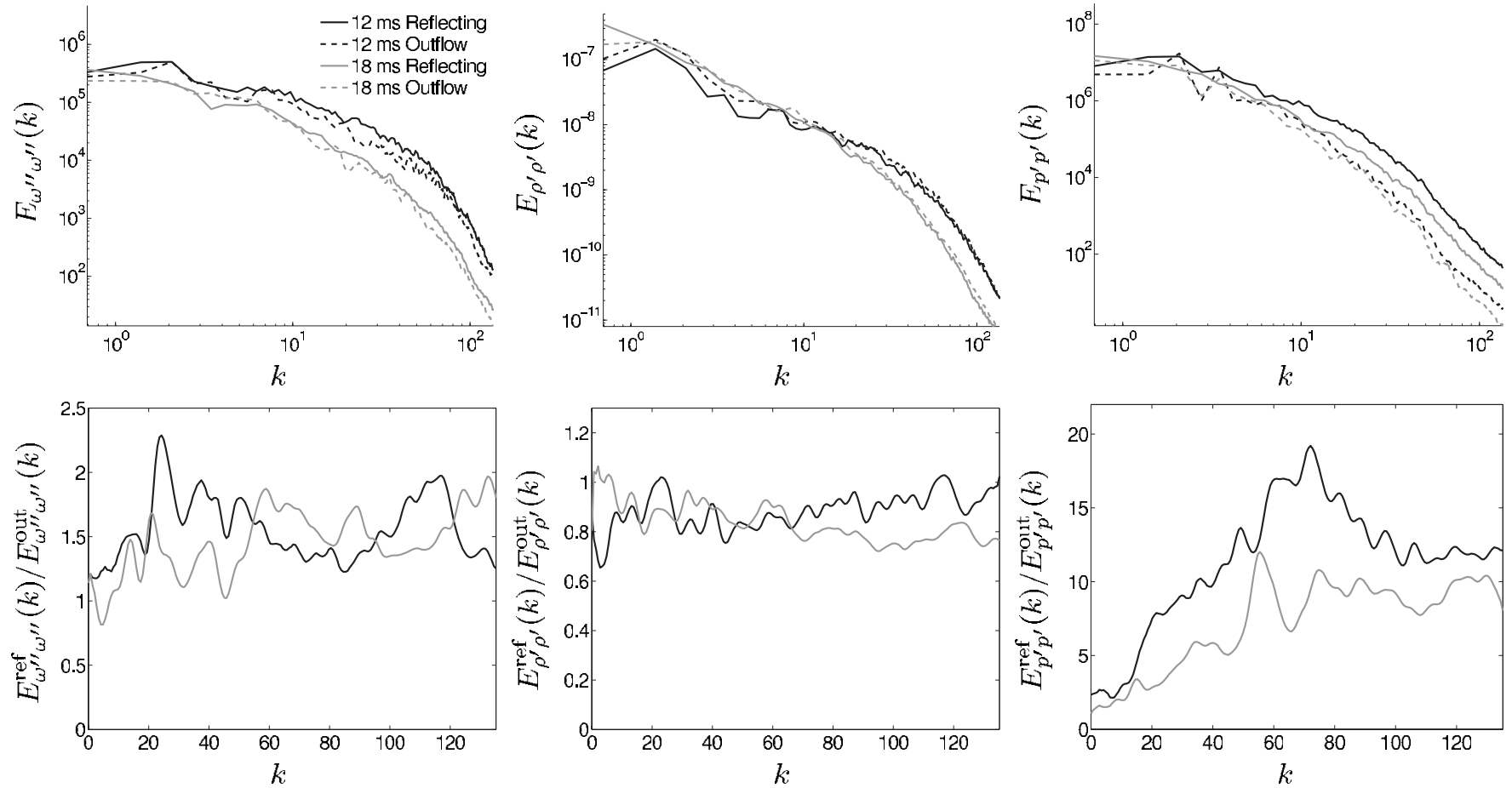

FIG. 29. Comparison of the fluctuating enstrophy spectrum $E_{\omega^{\prime \prime} \omega^{\prime \prime}}(k)$ (in units of $\mathrm{cm} / \mathrm{s}^{2}$ ), density variance spectrum $E_{\rho^{\prime} \rho^{\prime}}(k)$ (in units of $\mathrm{g}^{2} / \mathrm{cm}^{5}$ ), and pressure variance spectrum $E_{p^{\prime} p^{\prime}}(k)$ (in units of $\mathrm{bar}^{2} \mathrm{~cm}$ ) at $12 \mathrm{~ms}$ (black) and $18 \mathrm{~ms}$ (gray) for reflecting and outflow boundary conditions (top row). The ratios of the spectra from the reflecting and outflow cases are also shown (bottom row).

"vortex-accelerated secondary baroclinic vorticity deposition" [19]. This secondary instability is responsible for the complex structures in the rollups and for additional vorticity deposition of opposite sign on the interface, decreasing the circulation. Reshock causes the deposition of additional vorticity of opposite sign, which is further amplified by the secondary baroclinic vorticity deposition. Following the interaction with the reflected rarefaction wave, complex structures form and the flow breaks symmetry, resulting in large asymmetry between the positive and negative circulations and a nonzero total circulation.

\section{The post-reshock mixing layer width}

The mixing layer width immediately following reshock was shown to be consistent with the linear growth predicted by the Mikaelian [26] and Charakhch'yan [28] reshock models. The Brouillette-Sturtevant model underestimated the growth at all times following reshock. At later times following reshock, the width is affected by waves interacting with the layer.

\section{Analysis of mixing and the physics of reshock}

Mixing was investigated by considering the time evolution of the (i) mole fraction, production fraction, and mixing fraction profiles to quantitatively assess mixing across the layer, (ii) fluctuating kinetic energy, fluctuating enstrophy, density variance, pressure variance, and baroclinic vorticity production variance spectra to determine the modal distribution of the fluctuations, (iii) spatially averaged mixing frac- tions to quantify the efficiency of mixing, and (iv) statistics to characterize the time evolution of energy.

\section{Mole fraction and mixing fraction profiles}

The mole and mixing fraction profiles quantify mixing along the shock propagation direction. The mole fraction profile measures the redistribution of mass within the mixing layer caused by the creation of bubble- and spikelike structures, and the mixing fraction profile characterizes the efficiency of mixing. Prior to reshock, the main mechanism affecting these profiles is the formation of the single bubble and spike, which roll up at late times creating a region of well-mixed fluid within. Single localized peaks formed in the mole fraction and mixing fraction profiles prior to reshock. Prior to reshock, the evolution of the mixing fraction profile indicated increased mixing toward the air (acetone) side, corresponding to the formation of the rollup with a well-mixed core.

During reshock, the layer is compressed by the passage of the shock [which continues into the air (acetone)] and is "pulled back" on the $\mathrm{SF}_{6}$ side by the reflected rarefaction. This effect was captured by the mixing fraction profile, which is larger on the air (acetone) side and smaller on the $\mathrm{SF}_{6}$ side. Reshock significantly increased the mixing fractions as the mixing layer was compressed.

Following reshock, the inversion process, followed by the late-time formation of small bubbles and spikes, contributed to the formation of highly irregular, topologically complex structure on the interface. These additional bubbles and spikes elongated the mixing layer, resulting in a nearly uniformly increasing mass distribution across the layer from the 

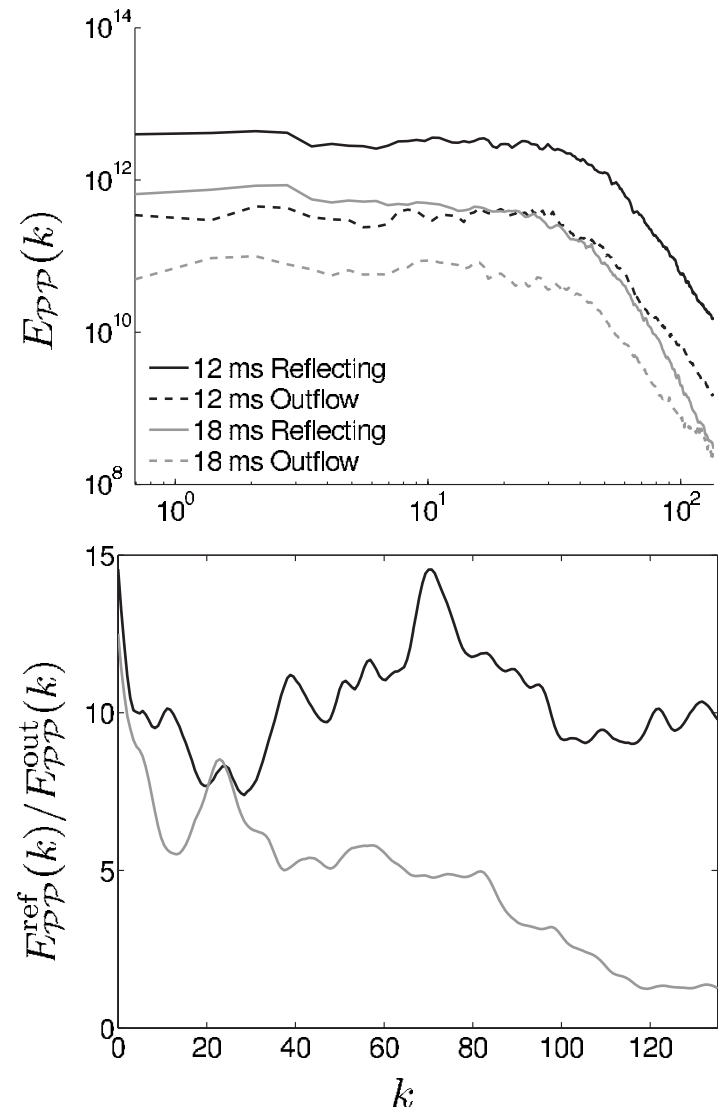

FIG. 30. Comparison of the baroclinic vorticity production variance spectrum $E_{\mathcal{P P}}(k, t)$ (in units of $\mathrm{cm} / \mathrm{s}^{4}$ ) at $12 \mathrm{~ms}$ (black) and $18 \mathrm{~ms}$ (gray) for reflecting and outflow boundary conditions (top). The ratio of the spectra from the reflecting and outflow cases is also shown (bottom).

air (acetone) to the $\mathrm{SF}_{6}$ side. Several smaller rollups also formed, indicating large regions of locally well-mixed air and $\mathrm{SF}_{6}$. The mixing fractions also showed that these regions grow larger at late times, corresponding to more complete mixing. This is consistent with the inverse cascade of smallscale velocity fluctuations to larger scales in twodimensional turbulence. The profiles indicated a late-time collapse towards self-similarity within the mixing layer.

\section{Production and mixing fractions}

The total chemical product, maximum chemical product, and global mixing fractions quantify the enhanced mixing caused by reshock. Before reshock, the total chemical product increased, indicating an increase in mixing. During reshock, the mixing layer is compressed, inducing additional mixing as measured by the total chemical product. Following reshock, the total chemical product increased rapidly, indicating significantly increased mixing. Immediately after reshock, the mixing fractions were sharply peaked. Following reshock, these fractions increased faster than before reshock, indicating that reshock increased the mixing rate.

\section{Energy spectra}

The spectral evolution prior to and following reshock provides a modal quantification of the complex features in the
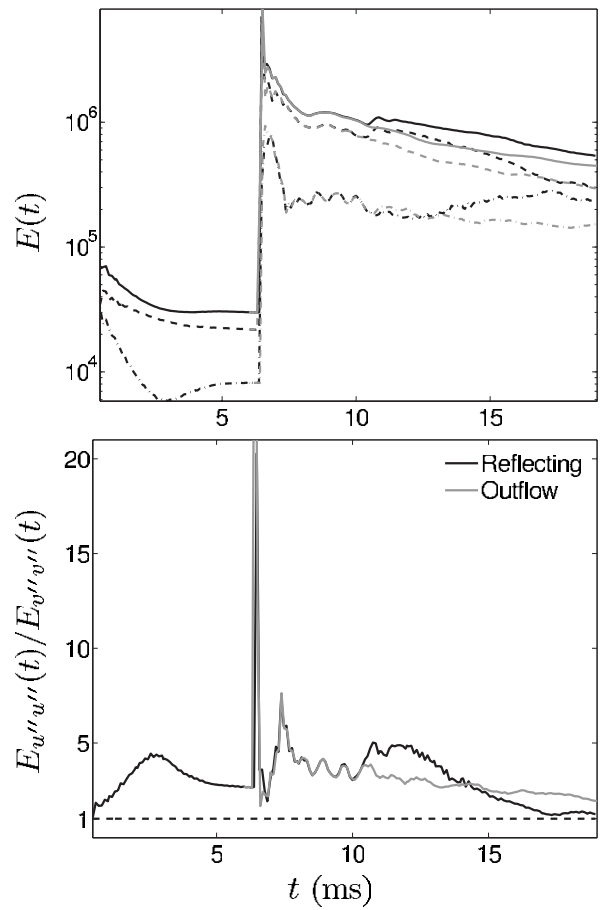

FIG. 31. Comparison of the time evolution of the fluctuating kinetic energy $E(t)$ (solid line) and its streamwise and spanwise components $E_{u^{\prime \prime} u^{\prime \prime}}(t)$ and $E_{v^{\prime \prime} v^{\prime \prime}}(t)$ (dashed and dot-dashed lines, respectively) (in units of $\mathrm{cm}^{2} / \mathrm{s}^{2}$ ) for reflecting and outflow boundary conditions (top). The time evolution of the ratio of the streamwise and spanwise component of the fluctuating kinetic energy $E_{u^{\prime \prime} u^{\prime \prime}}(t) / E_{v^{\prime \prime} v^{\prime \prime}}(t)$ is also shown (bottom).

density, vorticity, and baroclinic vorticity production fields. It was shown that a broad range of scales already exists prior to reshock. At reshock, the fluctuations in all fields (except for the density) were amplified across all scales. Reshock does not appear to broaden the range of scales, but primarily imparts energy into structures of all sizes. At late times, numerical dissipation smooths the spectra. When the shock has moved far away from the layer and the reflected waves become weaker, the spectra decay very slowly.

The decomposition of the fluctuating kinetic energy spectrum into its streamwise and spanwise components indicated that the former is dominant, and that significant spectral anisotropy in the velocity field exists even at late times. Compared to the fluctuating kinetic energy spectrum, the fluctuating enstrophy spectrum exhibited a more pronounced cascade toward smaller scales, as expected from twodimensional turbulence phenomenology. The pressure variance spectrum increased following reshock and during the arrival of the reflected rarefaction. The density variance spectrum showed that density fluctuations remain small throughout the flow evolution and are not strongly affected by reshock.

The possible emergence of inertial subrange scalings of the fluctuating kinetic energy, fluctuating enstrophy, density variance, and pressure variance spectra was also investigated by instantaneously comparing to the scaling predictions for two-dimensional turbulence using compensated spectra. Shortly after reshock $(8 \mathrm{~ms})$, the compensated spectra 

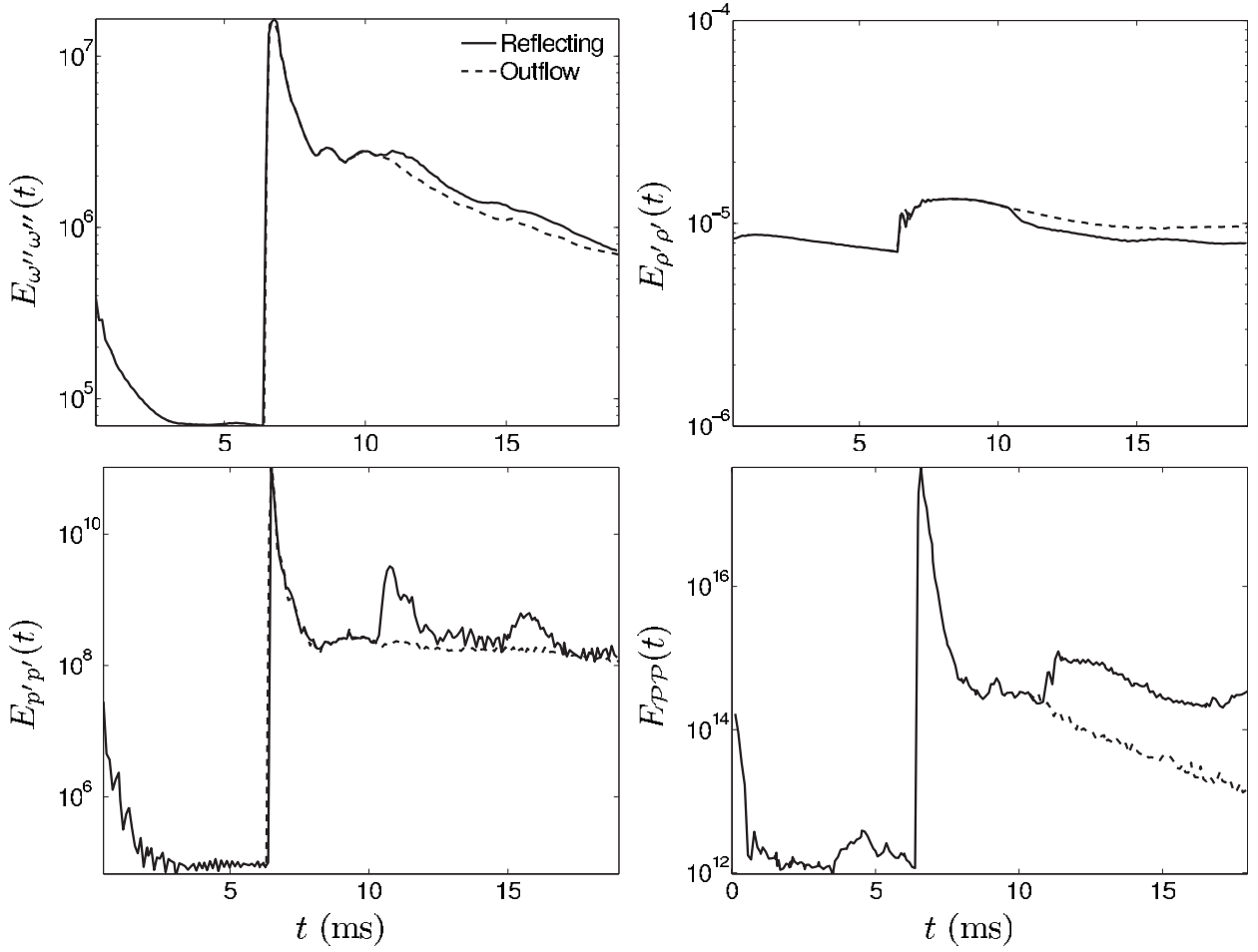

FIG. 32. Comparison of the time evolution of the fluctuating enstrophy $E_{\omega^{\prime \prime} \omega^{\prime \prime}}(t)$ (in units of $\mathrm{s}^{-2}$ ), density variance $E_{\rho^{\prime} \rho^{\prime}}(t)$ (in units of $\left.\mathrm{g}^{2} / \mathrm{cm}^{6}\right)$, pressure variance $E_{p^{\prime} p^{\prime}}(t)$ (in units of $\mathrm{bar}^{2}$ ), and baroclinic vorticity production variance $E_{\mathcal{P P}}(t)$ (in units of $\mathrm{s}^{-4}$ ) for reflecting and outflow boundary conditions (solid and dashed lines, respectively). showed that the turbulence induced by the reshocked Richtmyer-Meshkov instability exhibits energy transfer both to smaller scales (forward cascade) and to larger scales (inverse cascade), and approximate inertial subranges may exist for short wave number extents. However, at late time (18 $\mathrm{ms})$ the fluctuating enstrophy spectra exhibited large and intermediate wave number scalings different from those expected from classical two-dimensional isotropic turbulence phenomenology, as a consequence of the transient baroclinic forcing mechanism in the reshocked Richtmyer-Meshkov instability. The density variance spectrum may exhibit an inertial-convective subrange scaling expected for a passive scalar, and the pressure variance spectrum may scale as expected in two-dimensional turbulence. However, an even larger range of scales must be simulated to definitively examine these putative scalings. Reshock is also necessary to attain the intensity of turbulence and to develop the broad range of scales necessary to achieve such scalings.

\section{Statistics}

The time evolution of statistics was also investigated. The fluctuating kinetic energy and enstrophy exhibited sharp peaks corresponding to reshock, followed by a rapid decrease, then followed by a decay. The decay becomes more established following the interaction with the reflected rarefaction. The streamwise and spanwise fluctuating kinetic energies indicated that statistical isotropy is approached at very late times. The spanwise fluctuating kinetic energy increased following the interaction with reflected waves and then decreased due to dissipation. The pressure variance showed narrow sharp peaks corresponding to reshock and to the arrival of the reflected rarefaction, and further showed the arrival of the reflected compression wave at late times. These waves excite fluctuations that are apparent in the increased values of the statistics. The fluctuations were damped and the variances decreased as time evolved. The density variance was very small and remained nearly constant, although signatures of the reflected shock and of the reflected rarefaction were evident.

\section{E. Comparison of mixing quantities and spectra immediately after and before reshock}

The comparison of mixing quantities and spectra immediately after and before reshock quantified the effects of reshock on the mixing layer and the energy deposition into the layer by baroclinic mechanisms. Reshock compressed the layer and shifted mass from the $\mathrm{SF}_{6}$ side toward the air (acetone) side, consistent with the direction of reshock from the heavier gas into the lighter gas. Reshock further generated structures within the mixing layer, creating a well-mixed region close to the $\mathrm{SF}_{6}$ and peaks closer to the air (acetone). This was also reflected by the mixing fraction $\theta$.

The energy deposition by reshock was quantified by examining the amplification of the energy spectra. Reshock amplified the fluctuating kinetic energy spectra most at larger wave numbers, consistent with the formation of small-scale, vortical structures following reshock. The pressure variance is amplified most by reshock, while the density variance is only slightly amplified, indicating that it does not have a significant role in the post-reshock flow dynamics. Thus the fluctuating kinetic energy, fluctuating enstrophy, and pressure variance spectra are useful for the characterization of the modal distribution of energy within the mixing layer following reshock. The amplifications are dependent upon the shock Mach number and on other details of the flow configuration. 


\section{F. The effects of reflected waves on mixing}

The effects of the reflected rarefaction wave on the mixing layer were also investigated through a comparison of quantities from simulations using reflecting and outflow boundary conditions. The reflected rarefaction did not significantly increase the energy content in the layer, but contributed to flow symmetry breaking and driving the flow towards statistical isotropy at late times by exciting fluctuations in the spanwise direction. The reflected rarefaction also had a small effect on the fluctuating enstrophy and pressure variance evolution. By contrast, reshock compressed the layer and caused significant deposition of energy that induced rapid growth of the layer, concomitant with the formation of complex structures. The reflected rarefaction instead "stretched" the mixing layer (causing it to grow), but provided little additional energy, so that the overall mixing decreased, as quantified by the mixing fractions. The additional mixing induced by the reflected waves was responsible for a faster approach to late-time statistical isotropy. This comparison further supports the important role that reflected waves have in the post-reshock flow dynamics. The interactions of the waves with the evolving layer can be interpreted as small, transient forcings at all scales.

\section{G. Final remarks}

The WENO shock-capturing method was used here to investigate the physics of reshock and mixing by computing and physically interpreting quantities not presently available from Richtmyer-Meshkov instability experiments. Specifically, a wide range of quantities that have been used to characterize other mixing layers were considered. A comparison of such quantities just after and before reshock quantified the effects of reshock, including the mechanisms of energy deposition. A comparison of these quantities for outflow and reflecting boundary conditions quantified the important role that successive wave-interface interactions have on mixing. Additional experimental data pertaining to the post-reshock phase, including measurements of quantities depending on spatial derivatives (vorticity or enstrophy) or quantities sensitive to small-scale mixing (mixing fractions), is needed to further validate numerical simulations and constrain models.

\section{ACKNOWLEDGMENTS}

M.L. acknowledges support from the Air Force Office of Scientific Research and the Caltech Accelerated Strategic Computing (ASC) Center. W.S.D. acknowledges support by the DOE under Contract No. DE-FG02-98ER25346 and the AFOSR under Contract No. FA9550-05-1-0123. This work was performed under the auspices of the U.S. Department of Energy by the University of California, Lawrence Livermore National Laboratory under Contract No. W-7405-Eng-48.
[1] B. D. Collins and J. W. Jacobs, J. Fluid Mech. 464, 113 (2002)

[2] M. Latini, O. Schilling, and W. S. Don, Phys. Fluids 19, 024104 (2007).

[3] W. D. Arnett, Astrophys. J. 319, 136 (1987).

[4] J. D. Lindl, Inertial Confinement Fusion: The Quest for Ignition and Energy Gain Using Indirect Drive (Springer-Verlag, New York, 1997).

[5] J. M. Taccetti, S. H. Batha, J. R. Fincke, N. D. Delamater, N. E. Lanier, G. R. Magelssen, R. M. Hueckstaedt, S. D. Rothman, C. J. Horsfield, and K. W. Parker, Astrophys. Space Sci. 298, 327 (2005).

[6] J. R. Rygg, Ph.D. thesis, Massachusetts Institute of Technology, 2006.

[7] D. L. Youngs, Laser Part. Beams 12, 725 (1994).

[8] C. Mügler and S. Gauthier, Phys. Rev. E 58, 4548 (1998).

[9] M. Latini, O. Schilling, and W. S. Don, J. Comput. Phys. 221, 805 (2007)

[10] D. Balsara and C.-W. Shu, J. Comput. Phys. 160, 405 (2000).

[11] S. Karni, J. Comput. Phys. 112, 31 (1994).

[12] S. Karni, SIAM J. Sci. Comput. 17, 1019 (1996).

[13] R. Abgrall, J. Comput. Phys. 125, 150 (1996).

[14] R. Abgrall and S. Karni, J. Comput. Phys. 169, 594 (2001).

[15] M. R. Meloon, Ph.D. thesis, California Institute of Technology, 1998.

[16] D. J. Hill, C. Pantano, and D. I. Pullin, J. Fluid Mech. 557, 29 (2006).

[17] P. J. Linstrom and W. G. Mallard, NIST Chemistry WebBook,
NIST Standard Reference Database No. 69 (National Institute of Standards and Technology, Gaithersburg, MD, 2005), http:// webbook.nist.gov

[18] N. Themelis, Transport and Chemical Rate Phenomena (Taylor and Francis, New York, 1995).

[19] G. Peng, N. J. Zabusky, and S. Zhang, Phys. Fluids 15, 3730 (2003).

[20] A. Marquina and P. Mulet, J. Comput. Phys. 185, 120 (2003).

[21] M. Brouillette and B. Sturtevant, Phys. Fluids A 5, 916 (1993).

[22] R. Samtaney and N. J. Zabusky, J. Fluid Mech. 269, 45 (1994).

[23] M. C. Soteriou and A. F. Ghoniem, Phys. Fluids 7, 2036 (1995).

[24] C. Staquet, J. Fluid Mech. 296, 73 (1995).

[25] J. Reinaud, L. Joly, and P. Chassaing, Phys. Fluids 12, 2489 (2000).

[26] K. O. Mikaelian, Physica D 36, 343 (1989).

[27] M. Brouillette and B. Sturtevant, Physica D 37, 248 (1989).

[28] A. A. Charakhch'yan, Plasma Phys. Controlled Fusion 43, 1169 (2001).

[29] K. I. Read, Physica D 12, 45 (1984).

[30] D. L. Youngs, Physica D 12, 32 (1984).

[31] M. Vetter and B. Sturtevant, Shock Waves 4, 247 (1995).

[32] R. E. Duff, F. H. Harlow, and C. W. Hirt, Phys. Fluids 5, 417 (1962).

[33] A. W. Cook and P. E. Dimotakis, J. Fluid Mech. 443, 69 (2001); 457, 410 (2002). 
[34] A. Favre, in Studies in Turbulence, edited by T. B. Gatski, S. Sarkar, and C. G. Speziale (Springer-Verlag, New York, 1992), pp. 324-341.

[35] P. Chassaing, R. A. Antonia, F. Anselmet, L. Joly, and S. Sarkar, Variable Density Fluid Turbulence, Fluid Mechanics and its Applications Vol. 69 (Kluwer Academic, New York, 2002).

[36] M. Lesieur, C. Staquet, P. Le Roy, and P. Comte, J. Fluid Mech. 192, 511 (1988).

[37] C. Mügler and S. Gauthier, Phys. Fluids 12, 1783 (2000).

[38] S. B. Pope, Turbulent Flows (Cambridge University Press, Cambridge, England, 2000).

[39] M. M. Koochesfahani and P. E. Dimotakis, J. Fluid Mech. 170, 83 (1986)

[40] D. L. Youngs, Phys. Fluids A 3, 1312 (1991).

[41] R. H. Kraichnan, Phys. Fluids 10, 1417 (1967).

[42] G. K. Batchelor, Phys. Fluids 12, 233 (1969).

[43] R. H. Kraichnan and D. Montgomery, Rep. Prog. Phys. 43, 547 (1980).

[44] M. Lesieur, Turbulence in Fluids, Fluid Mechanics and its Applications Vol. 40, 3rd ed. (Kluwer Academic, Amsterdam, 1997).

[45] J. Sommeria, in New Trends in Turbulence, Les Houches Sum- mer School on Theoretical Physics LXXIV, 2000, edited by M. Lesieur, A. Yaglom, and F. David (Springer-Verlag, Berlin, 2001), pp. 385-447.

[46] P. Tabeling, Phys. Rep. 362, 1 (2002).

[47] R. H. Kraichnan, J. Fluid Mech. 47, 525 (1971).

[48] A. M. Obukhov, Izv. Akad. Nauk SSSR, Ser. Fiz. 13, 58 (1949).

[49] S. Corrsin, J. Appl. Phys. 22, 469 (1951a).

[50] S. Corrsin, J. Aeronaut. Sci. 18, 417 (1951b).

[51] G. K. Batchelor, J. Fluid Mech. 5, 113 (1959).

[52] A. S. Monin and A. M. Yaglom, Statistical Fluid Mechanics (MIT Press, Cambridge, 1971), Vol. 1.

[53] M. Lesieur, S. Ossia, and O. Métais, Phys. Fluids 11, 1535 (1999).

[54] J. W. Jacobs and V. V. Krivets, Phys. Fluids 17, 034104 (2005).

[55] Y. Andreopoulos, J. H. Agui, and G. Briassulis, Annu. Rev. Fluid Mech. 32, 309 (2000).

[56] G. Briassulis, J. H. Agui, and Y. Andreopoulos, J. Fluid Mech. 432, 219 (2001).

[57] J. H. Agui, G. Briassulis, and Y. Andreopoulos, J. Fluid Mech. 524, 143 (2005). 